\title{
Structural features of Dnase1L3 responsible for serum antigen clearance
}

\author{
Jon J. McCord ${ }^{1}$, Minal Engavale ${ }^{2}$, Elahe Masoumzadeh ${ }^{3}$, Johanna Villarreal ${ }^{1}$, Britney \\ Mapp $^{2}$, Michael P. Latham ${ }^{3}$, Peter A. Keyel ${ }^{2}$, and R. Bryan Sutton ${ }^{1}$
}

\author{
${ }^{1}$ Texas Tech University Health Sciences Center, Dept of Cell Physiology and Molecular Biophysics \\ ${ }^{2}$ Texas Tech University, Dept. of Biological Sciences \\ ${ }^{3}$ Texas Tech University, Dept. of Chemistry \& Biochemistry
}

\begin{abstract}
Autoimmunity develops when extracellular DNA released from dying cells is not cleared from serum. While serum DNA is primarily digested by Dnase1 and Dnase1L3, Dnase1 does not rescue autoimmunity arising from Dnase1L3 deficiencies. Dnase1L3 uniquely degrades antigenic forms of cell-free DNA, including DNA complexed with lipids and proteins. The distinct activity of Dnase1L3 relies on its unique C-terminal Domain (CTD), but the mechanism is unknown. We used multiple biophysical techniques and functional assays to study the interplay between the core catalytic domain and the CTD. While the core domain resembles Dnase1, there are several key differences between the two enzymes. Dnase1L3 is not inhibited by actin due to multiple differences in the actin recognition site. The CTD augments the ability of the core to bind DNA, thereby facilitating the degradation of complexed DNA to prevent autoimmune pathology. Together, these structural insights will inform the development of Dnase1L3-based therapies for autoimmunity.
\end{abstract}

\section{Main}

Production of antibodies targeting cell-free nucleic acids can lead to the development of severe autoimmune disorders, including systemic lupus erythematosus (SLE) ${ }^{1}$, hypocomplementemia urticarial vasculitis syndrome (HUVS) ${ }^{2}$, and rheumatoid arthritis $(\mathrm{RA})^{3}$. The enzymes responsible for clearing antigenic DNA from serum have been identified as Dnase1 and Dnase1L3 (deoxyribonuclease 1 like 3 or Dnase $\gamma)^{4,5}$; yet, the activities assigned to these two enzymes are not synonymous. For example, mutations in Dnase1L3 have been linked to multiple autoimmune diseases ${ }^{2,6-8}$, while Dnase1 is unable to rescue these autoimmune phenotypes ${ }^{6,7}$. Therefore, Dnase1L3 accounts for a broader range of nucleic acid degradative activities.

Based on the overall similarity between the two enzymes, the catalytic activities of Dnase1 and Dnase1L3 should be similar. Dnase 1 and Dnase1L3 are both $\mathrm{Ca}^{2+} / \mathrm{Mg}^{2+}$-dependent endonucleases with a high degree of identity in the active site; however, the catalytic mechanism of the Dnase1-family remains contested ${ }^{9}$. Currently, there are two leading models for Dnase 1 family catalysis, the single cation carboxylic acid model ${ }^{10}$ and double divalent cation model ${ }^{11}$. The principal differences between the proposed mechanisms are the number of divalent cations involved in catalysis, and the residues that directly participate in acid/base catalysis. The more recent single cation carboxylic acid model posits a single divalent cation and Asp-189 as the catalytic base ${ }^{10,12}$. In contrast, the more established double divalent cation mode $1^{13}$ predicts two cations and implicates His-155 as the catalytic acid and His-274 as the catalytic base ${ }^{11}$; however, neither model is completely supported by experimental data.

While the catalytic mechanism may be similar, there are multiple functional differences between Dnase1 and Dnase1L3. Dnase1 is inhibited by actin, while Dnase1L3 is not ${ }^{14}$. Furthermore, Dnase1L3 degrades DNA complexed with proteins and lipids, including immune complexes, more efficiently than Dnase 1 6,8,15,16. This unique Dnase1L3 activity is hypothesized to originate from its positively charged C-terminal domain (CTD) that extends from Ser- 283 to Ser- $305^{6}$. Previous homology modeling, including models generated by AlphaFold ${ }^{17}$ predicts that the CTD forms a rigid $\alpha$-helix, albeit with low confidence, which projects out from the core enzyme. It has been suggested that the C-terminal $\alpha$-helix facilitates membrane binding, penetration or could potentially displace bound histones that occlude the exposure of substrate DNA ${ }^{6}$.

Here we investigated the structure of the Dnase1L3 core domain using X-ray crystallography and the isolated CTD using Nuclear Magnetic Resonance (NMR) spectroscopy. The two domains were combined using Small-Angle X-ray Scattering (SAXS). Our high-resolution crystal structure of Dnase1L3 provides the first experimental support for the two-cation catalytic mechanism, wherein His-155 and His-274 act as the catalytic acid and base residues, respectively. The functional effects of the 
CTD were studied using enzymatic assays, co-sedimentation, and fluorescence polarization. We tie these functional assays to structural features of the CTD with circular dichroism and molecular dynamics to present a model of Dnase1L3's diverse DNA degradation capacity and the function of the intrinsically disordered CTD. Our results support a DNA binding role for the CTD that confers increased avidity to heterogeneous DNA.

\section{Results}

\section{MBP fusion enables prokaryotic expression of active Dnase1L3}

Dnase1 and Dnase1L3 are nonspecific endonucleases that induce chromosomal DNA laddering. The DNA digesting activity of Dnases complicated previous attempts to overproduce the enzyme using prokaryotic expression systems ${ }^{18}$; however, the expression of inactive Dnase 1 mutants has been successful ${ }^{19}$. To produce active Dnase1L3 in bacteria, we expressed the enzyme as a $\mathrm{His}_{6}$-Maltose Binding Protein (MBP)-Dnase1L3 fusion protein. The fusion protein approach facilitates the purification of active enzyme as the Dnase1L3 fusion is inactive prior to TEV cleavage of MBP from Dnase1L3 (Supplementary Figure S1-A). Prokaryotic expression offers a higher yield and a more straightforward workflow compared to the current eukaryotic expression protocols ${ }^{20}$.

\section{Structure of the core enzyme of Dnase1L3}

Full-length Dnase1L3 was refractory to crystallization, likely because of the flexible C-terminus; therefore, we deleted the last 23 residues of Dnase1L3 ( $\triangle$ CTD) for the structural analysis of the core domain. Dnase1L3 $\Delta$ CTD was crystallized at pH 8.5 in $250 \mathrm{mM} \mathrm{MgCl}_{2}$ in the P1 triclinic space group with four Dnase1L3 $\Delta$ CTD molecules in the asymmetric unit. The core Dnase1L3 enzyme was refined to a final resolution of $2.2 \AA$ with an R-free of $23 \%$ (Supplementary Table S1). The crystal structure of Dnase1L3 exhibits the classic 4-layer $\alpha-\beta$ sandwich architecture similar to other members of the exo/endo phosphatase (EEP) family of enzymes. The Dnase1L3 core enzyme structure superimposes with human Dnase $1^{10}$ with an RMSD of $1.8 \AA$ across all C- $\alpha$ atoms (Supplementary Figure S2-A). Post-translationally Dnase1 possesses two N-linked glycosylation modifications that are implicated in Dnase1 stabilization and wild-type nuclease activity ${ }^{21}$; however, the N-linked glycosylation sites at Asn-18 and Asn-106 on Dnase1 correspond to Asp residues (Asp-38 and Asp-126) in human Dnase1L3 (Supplementary Figure S2-B,C).

\section{Multifactorial actin resistance of Dnase1L3}

Dnase1 activity is inhibited by G-actin, DNA-actin complexes, and DNA in aggregates with other poly-cations ${ }^{23}$. Consequently, native Dnase1 as a therapy for cystic fibrosis $(\mathrm{CF})$ pulmonary sputum fluidization is limited. Dnase1 was reengineered as Pulmozyme ${ }^{\circledR}$ to minimize actin inhibition, but there remains a weak inhibition of Dnase1 by large DNA/actin complexes in CF patient sputum ${ }^{24}$. Dnase1L3 is natively resistant to actin inhibition ${ }^{14}$, making this enzyme a valuable therapeutic target. We compared Dnase1L3 with the Dnase1-actin crystal structure ${ }^{22}$ to elucidate the differences in the actin-binding interface. There are four notable differences between the two enzyme interfaces that directly impact actin binding. First, the 'central hydrophobic' region that Dnase 1 utilizes for actin binding is sterically disrupted in Dnase1L3 ${ }^{25}$. Dnase 1 actin resistance was experimentally conferred by mutating Ala-114 within the 'central hydrophobic region' to a bulky residue such as Arg, Glu, Met, Tyr, or Phe ${ }^{23}$. In Dnase1L3, the homologous residue to Ala-114 is Phe-131, which contributes to its native actin resistance (Figure 1-A). Second, a two amino acid insertion in the loop (residues 75-85 in Dnase1L3) following the actin-binding $\alpha$-helix creates a more flexible loop in Dnase1L3, with an inverted net charge, relative to Dnase1 (residues 55-63), from a pI of 3.45 in the actin-inhibited Dnase1 structures ${ }^{22}$ to $\mathrm{pI}$ of 11.15 in the actin-resistant Dnase1L3 (Figure 1-B). The charge inversion in the homologous actin-associated loop likely repels the similarly charged actin from productively binding to Dnase1L3. Third, an important salt bridge forming residue for Dnase1-actin binding is Glu-69 in Dnase1, which associates with Lys-61 from actin $^{22}$. In Dnase1L3, Glu-69 is analogous to Ser-91 (Supplementary Figure S3), thus eliminating the potential for a salt-bridge to actin. Finally, the actin-binding $\alpha$-helix (residues 67-75) is 'out-of-register' in our crystal structure of Dnase1L3 relative to the homologous $\alpha$-helix in Dnase1 (Figure 1-C). It is possible that this 'out-of-register' shift disrupts a critical salt-bridge for actin binding.

\section{The patient mutation R206C disrupts a critical salt-bridge network}

The best characterized pathogenic mutation in Dnase1L3 is the hypofunctional R206C that reduces enzyme activity and protein secretion $^{26,27}$. Using our crystal structure as a starting point, we performed 500 ns molecular dynamics simulations in explicit solvent equilibrated in the presence of $150 \mathrm{mM} \mathrm{MgCl}_{2}$ on Chain $\mathrm{C}$ of Dnase1L3 $\Delta \mathrm{CTD}$ mutated to R206C. In addition to the previously predicted Glu-170 to Arg-206 salt bridge ${ }^{28}$, our structural analysis shows that Arg-206 forms an extensive salt-bridged network with Asp-166 and Glu-167. The crystal structure shows well-ordered electron density for the Arg-206 residue, as well as the salt-bridged Asp-166 residue (Supplementary Figure S4). Glu-167 and Glu-170 are likely transiently salt-bridged with Arg-206 because their electron density is not well-defined. The triple salt-bridged Arg-206 residue 

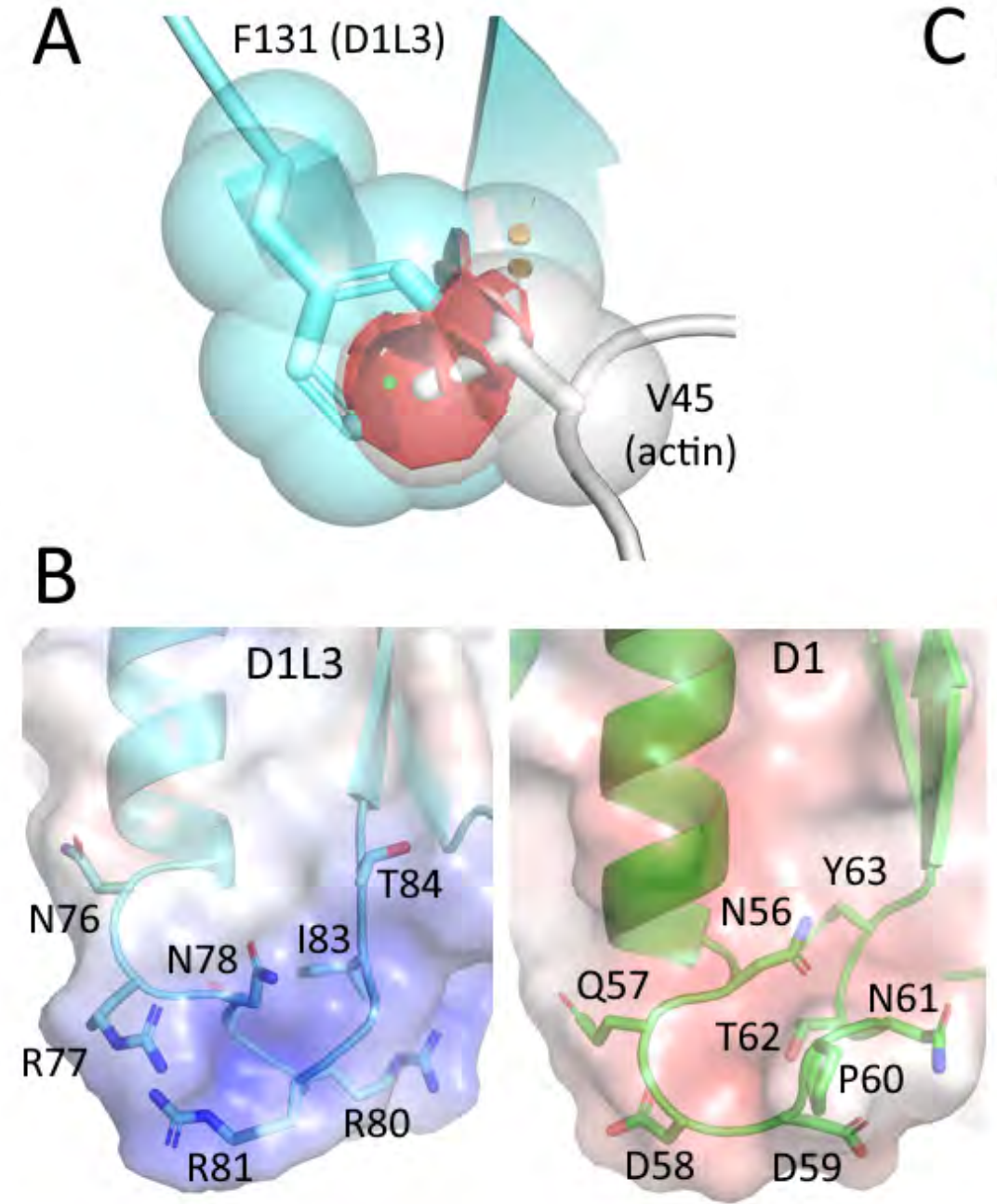

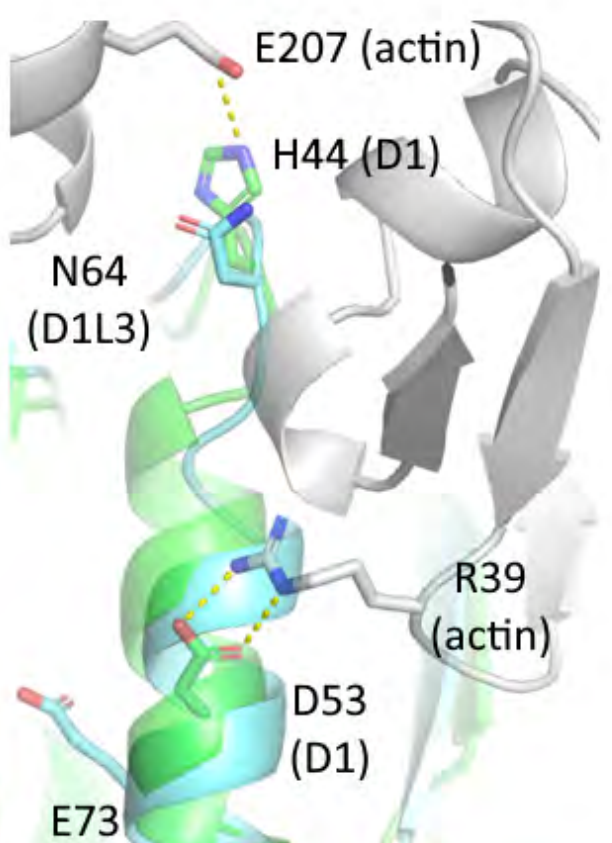

(D1L3)

Figure 1. Dnase1L3 inhibits actin binding: A) Dnase1L3 (blue) possesses a Phe-131 at the Ala-114 actin-binding locus in Dnase1 that clashes (in red) with Val-45 of actin (grey) when docked ${ }^{22}$. B) Dnase1 (green) is acidic (pI=3.45) at the actin interface, whereas Dnase1L3 (blue) is basic ( $\mathrm{pI}=11.15$ ) at the homologous site. C) The salt bridge interactions from Dnase1 (green) to actin (grey) are disrupted in Dnase1L3 due to the pertinent helix ( residues 67-75) from Dnase1L3 (blue) being out of register relative to Dnase1.

is, therefore, a key stabilizing residue in the Dnase1L3 molecule. The R206C mutation eliminates the salt-bridge network (Supplementary Figure S5-A). Based on molecular dynamics simulation and Rosetta energy scoring ${ }^{29}$, the loss of the salt bridge network increases $\Delta \Delta \mathrm{G}$ of folding by $14 \mathrm{kcal} / \mathrm{mol}$ (Supplementary Figure S5-B,C). The reduced structural stability of R206C can cause misfolding, thereby reducing catalytic activity and impairing secretion to account for the net hypofunctionality of the R206C mutation.

\section{A single structural divalent cation and a unique disulfide in the core enzyme}

All members of the Dnase enzyme family require both structural and catalytic divalent cations. Dnase 1 crystal structures ${ }^{10,22}$ show evidence for four $\mathrm{Ca}^{2+}$ or $\mathrm{Mg}^{2+}$ binding sites, but a total of five cations have been postulated ${ }^{30}$. In Dnase1L3, we observed three divalent cations, with two $\mathrm{Mg}^{2+}$ present within the catalytic site (Figure 2-A). Using the nomenclature from Guéroult ${ }^{30}$, the 'II' and 'III' structural cations are absent in Dnase1L3. In Dnase1, the 'II' divalent cation binding site is stabilized by a nearby disulfide bridge (Cys101-Cys104). The divalent cation coordinating residues in the 'II' binding site are conserved between Dnase1 and Dnase1L3, but the divalent cation binding site in Dnase1L3 is missing both disulfide cysteines and is one residue shorter relative to the homologous Dnase1 site (Figure 2-B). The missing disulfide bridge in Dnase1L3 prevents the coordinating residues from folding into the consensus divalent cation binding site. The lack of the 'II' cation binding site in 
Dnase1L3 increases the flexibility of the corresponding loop. Indeed, the B-factors are elevated between the site 'II' loops across each chain in the Dnase1L3 asymmetric unit (Supplementary Figure S6). Importantly, the absence of site 'II' does not disrupt the position of the key DNA binding residue Arg-132 (Arg-111 in Dnase1) ${ }^{19}$.

Cation coordination of the 'III' site is disrupted in Dnase1L3 because it lacks a critical Ca ${ }^{2+}$ coordinating residue. The primary $\mathrm{Ca}^{2+}$ coordinating residue in Dnase1, Asp-172, corresponds to Gly-193 in Dnase1L3 (Figure 2-C). The conserved conformation of the catalytic residue Asn-191 (Asn-170 in Dnase1) demonstrates that the Dnase1L3 Cys194-Cys231 disulfide bridge may be sufficient for active site stability, even without $\mathrm{Ca}^{2+}$ coordination at site 'III'. Additionally, Dnase1L3 includes an extra Cys24-Cys52 disulfide bond, corresponding to Ala-4 and Tyr-32 in Dnase1, respectively. The additional disulfide bridge in Dnase1L3 may partially compensate for the lack of two structural divalent cation sites.
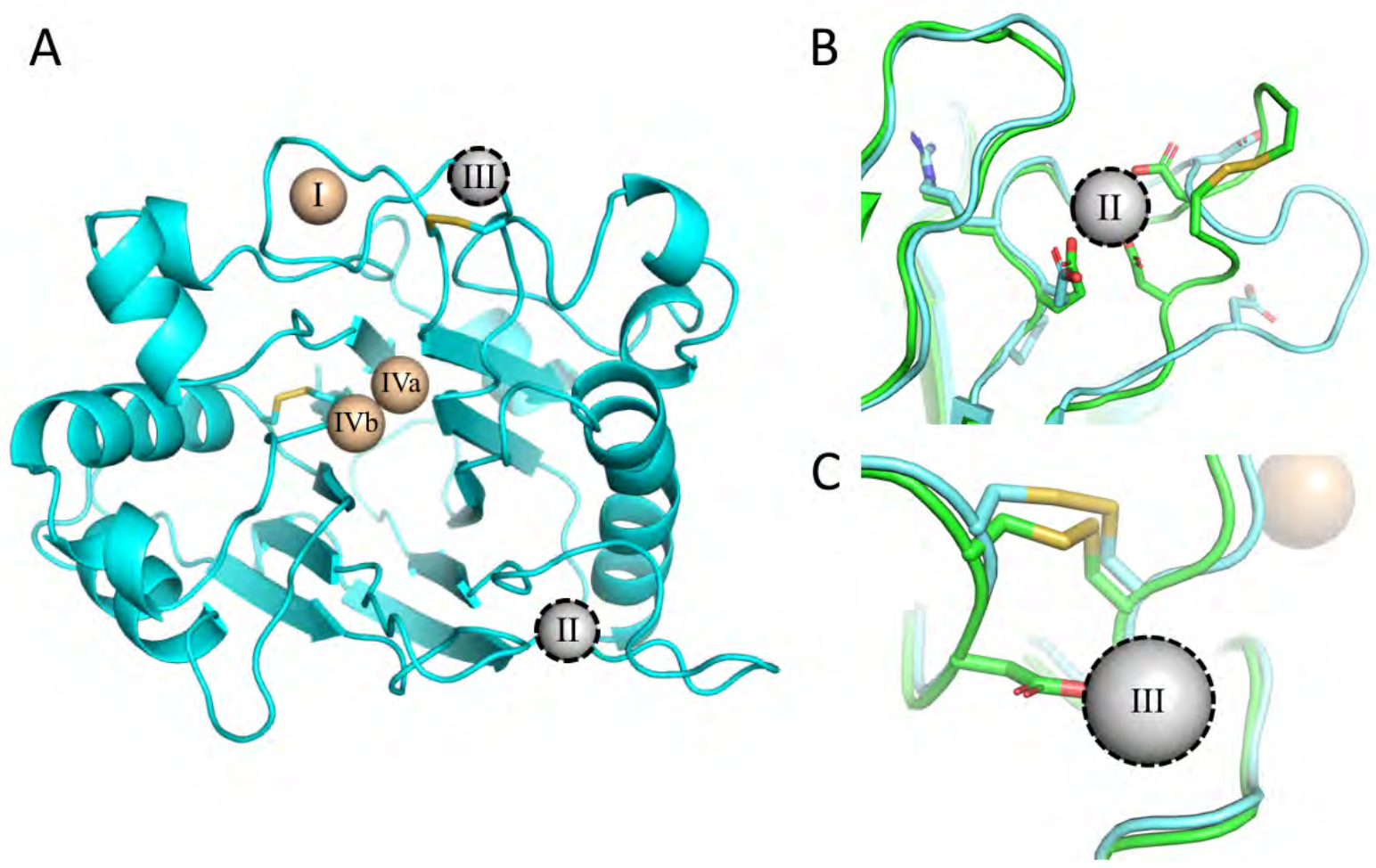

Figure 2. Dnase1L3 lacks structural divalent cations present in Dnase1: A) An overview of the divalent cations previously observed in the Dnase1 family superimposed onto the Dnase1L3 structure ${ }^{10,22}$. Metal ions colored tan were observed in the Dnase1L3 crystal structure. The outlined metal ions in grey are absent in Dnase1L3, but previously observed in Dnase1. B) View of missing structural divalent cation site 'II', observed in previous Dnase1 crystal structures ${ }^{22}$. Dnase1L3 is missing a disulfide bridge that stabilizes the loop structure of site 'II'. C) View of missing structural divalent cation site 'III', observed in previous Dnase1 (green) crystal structures ${ }^{22}$. The primary coordinating residue in Dnase1, Asp-172, is a Gly-193 in Dnase1L3 (blue).

\section{Structural evidence for the double divalent cation mechanism of Dnase1 family catalysis}

The two leading models of Dnase1-mediated DNA catalysis are the single-cation carboxylic acid and the double divalent cation model $^{9-11}$. The acidic crystallographic conditions of all previous structures precluded corroboration of either proposed model with respect to catalytic divalent cations ${ }^{10,22,32-37}$. Dnase1L3 crystallized with two $\mathrm{Mg}^{2+}$ in the active site (Supplementary Figure S7), which provides the first structural evidence of the mutagenesis-based double divalent cation model for Dnase1 family catalysis ${ }^{11}$ (Figure 3-A,B). To examine the interactions of the active site in the context of substrate DNA, we used all-atom molecular dynamics. We modeled DNA into the active site of Dnase1L3 based on the structure of Dnase1 crystallized with nucleic acid ${ }^{32}$. In our MD simulations, the two active site $\mathrm{Mg}^{2+}$ ('IVa' and 'IVb') each coordinate the oxygen atoms (OP1 and OP2) of the scissile phosphate of the DNA substrate. We observed a potential deprotonatable water coordinated by the 'IVb' divalent cation (Figure 3-C). Furthermore, His-155 and His-274 are in position for classical acid-base catalysis ${ }^{11}$ (Figure 3-C). The catalytic residue proposed in the single cation carboxylic acid model, Asp-189 (Asp-168 Dnase1), coordinates the 'IVa' divalent cation in our crystal structure and simulations (Figure 3-A,C). We tested the potential of each of the active site 


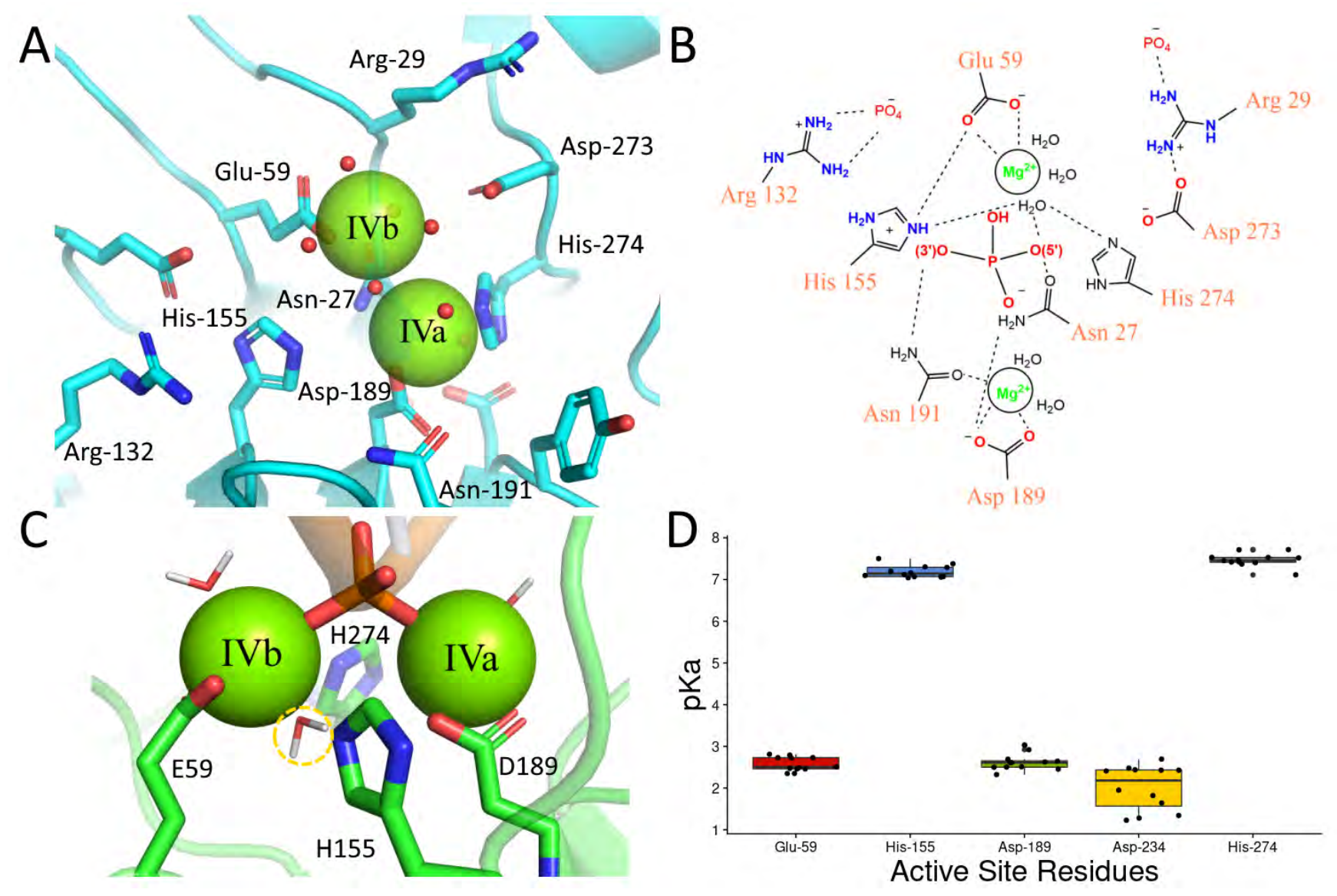

Figure 3. The Dnase1L3 catalytic site uses two divalent cations: A) Chain C of Dnase1L3 crystal structure active site showing both divalent cations in the active site. B) Schematic of the Dnase1L3 active site, based on the crystal structure and molecular dynamics, indicates the roles for key active site residues. C) Molecular dynamics simulation of Dnase1L3 structure in the presence of dsDNA. A potential deprotonatable water (highlighted in yellow) is coordinated by both His-155 and His 274 and positioned for backside attack of the scissile phosphate consistent with the SN2 reaction ${ }^{31}$. The scissile phosphate is coordinated by both divalent cations, and the active site His residues are positioned for acid-base catalysis. D) The acid dissociation constant for active site residues (with Dnase1L3 numbering) calculated from crystal structures of Dnase1 and Dnase1L3 $3^{10,22,32-37}$.

residues for acid-base catalysis via in silico $\mathrm{pH}$ titration of Dnase1L3 and Dnase1 (see Supplementary Methods). Calculations of the pKa of Asp-189 from the available Dnase1 structures and the four chains of our Dnase1L3 crystal structure indicate that Asp-189 maintains an acidic pKa (Figure 3-D), which predicts that Asp-189 cannot accept a proton during neutral pH acid-base catalysis. His-155 and His-274 are better candidates for acid-base catalytic residues, as their calculated pKa's are near neutral $\mathrm{pH}^{38}$ (Figure 3-D). Therefore, we propose Asp-189 is a divalent cation coordinating residue instead of a catalytic base.

The mechanism by which the putative catalytic acid, His-155, is regenerated for its next round of catalysis has not previously been determined. In our model, the regeneration of His-155 can be accomplished by an Arg-132 substrate-sensing switch. In the Dnase1L3 crystal structure, which lacks DNA, Arg-132 interacts with His-155 to decrease the pKa of His-155 favoring the deprotonated form (Supplementary Figure S8-A). Based on the MD simulation, in the presence of substrate DNA, Arg132 switches to bind to the DNA backbone; thus permitting His-155 to accept a proton that can then be donated to the 3'O (Supplementary Figure S8-B). Our Arg-132 switch hypothesis is supported by previous mutagenesis where the alanine substitution of the Arg-132 homolog in Dnase1 is catalytically inactive ${ }^{19}$. Our model predicts this mutation inactivates the enzyme by preventing His-155 regeneration. Another key residue identified by mutagenesis, Asn- $191^{19}$, coordinates the 'IVa' divalent cation in the Dnase1L3 crystal structure and MD simulations (Supplementary Figure S8-C,D). In our MD simulations with DNA, the $\delta 2$ amine of Asn-191 is positioned within hydrogen-bonding distance to the 3'O of the scissile phosphate of DNA (Supplementary Figure S8-D). Asn-191 H-bonding to the 3'O can stabilize the leaving group oxygen for nucleophilic 
attack by the protonating His-155 (Figure 3-B). We propose a new role for Asn-191 as both a 'IVa' cation coordinating and 3' ester stabilizing residue in accordance with previous mutagenesis results ${ }^{19}$. Our biophysical findings are consistent with the double divalent cation model of Dnase 1 catalysis ${ }^{11}$.

\section{The CTD promotes Dnase1L3 specific activity}

The CTD of Dnase1L3 is a 23 amino acid long, highly basic polypeptide unique to Dnase1L3. The CTD has previously been shown to confer increased degradative capacity on lipid complexed DNA ${ }^{6}$. To determine whether the CTD itself possesses inherent nuclease activity, we cloned the CTD of Dnase1L3 onto an unrelated carrier protein, the SH3 domain from the yeast protein, Abp1. We selected the Abp1 SH3 domain because it is well defined by NMR spectroscopy ${ }^{39}$, and it can aid in the overexpression of recombinant CTD polypeptide. We measured the Dnase1 activity of the purified SH3-CTD fusion protein and the SH3 domain alone and found that neither SH3 nor SH3-CTD degraded DNA (Supplementary Figure S1-B,C). To measure the effectiveness of the CTD in conjunction with the core Dnase1L3 enzyme, we quantitated the plasmid degradation activity of Dnase1, Dnase1L3, and Dnase1L3 $\Delta$ CTD. Recombinant full-length and Dnase1L3 $\Delta$ CTD had a 10-fold lower EC 50 , and therefore a higher relative activity, compared to standardized, commercially prepared Dnase 1 at the same plasmid substrate concentration (Figure 4-A). Because $\triangle$ CTD and full-length Dnase1L3 have comparable activity, we conclude that the increased naked plasmid degradation activity of Dnase1L3 relative to Dnase1 is not due to the CTD.

In contrast, the CTD from Dnase1L3 is necessary for Dnase1L3-specific activity against complexed DNA. We determined the contribution of the CTD to the degradation of lipid-complexed or antibody-bound DNA by testing full-length Dnase1L3 and Dnase1L3 $\triangle$ CTD with two Dnase1L3 assays: the barrier to transfection assay ${ }^{16}$ and an immune complex (IC) degradation assay. Full-length Dnase1L3 reduced transfection of HEK cells to a greater degree than Dnase1 or Dnase1L3 $\triangle$ CTD (Figure 4-B). Next, we tested Dnase1L3-specific activity against a more physiological DNA substrate, pathogenic autoantibody-DNA ICs. To determine the impact of the CTD on disrupting ICs, we measured chromatin immune complex degradation by ELISA. The ICs were treated with full-length Dnase1L3, Dnase1L3 $\Delta$ CTD, or Dnase1. Antibody-bound chromatin was most efficiently degraded by full-length Dnase1L3 (Figure 4-C). We measured a 4-fold improvement in the EC 50 of Dnase1L3 with ICs compared to Dnase1 and Dnase1L3 $\triangle$ CTD (Figure 4-D). Thus, the CTD of Dnase1L3 mediates the enhanced degradation of DNA in lipid- and antibody complexes, but not naked plasmid DNA.

\section{The CTD of Dnase1L3 is a disordered DNA binding domain}

The mechanism by which the CTD confers Dnase1L3-specific activity remains unknown. One hypothesis is that the Dnase1L3 CTD forms a C-terminal $\alpha$-helix that binds and penetrates microparticles and lipids to liberate DNA from DNA-lipid complexes ${ }^{6}$. We tested this hypothesis by incubating full-length Dnase1L3 and Dnase1L3 $\Delta$ CTD with pre-defined liposomes (20:20:10:50/PC:PE:PS:cholesterol) and performed a co-sedimentation assay. Surprisingly, Dnase1L3 $\Delta$ CTD bound lipids with higher avidity compared to full-length Dnase1L3 (Figure 4-E). Furthermore, neither SH3-CTD nor SH3 alone bound phospholipids (Figure 4-E). Next, we tested whether the CTD bound to microparticles purified from HeLa cells. Similar to our results with liposomes, the CTD decreased microparticle interaction (Figure 4-F). Therefore, we conclude that the CTD does not participate in lipid binding.

We next determined the ability of the CTD to interact with DNA. To experimentally determine DNA binding, the CTD was cleaved from the SH3-CTD fusion protein using TEV protease and then purified away from the SH3 and TEV. DNA binding experiments were performed using the purified CTD peptide titrated into fluorescently-labeled 40-mer dsDNA in $100 \mathrm{mM}$ $\mathrm{NaCl}$ at $\mathrm{pH}$ 7.4. In the fluorescent anisotropy assay, reduced tumbling of the dsDNA indicated CTD binding. The resulting $\mathrm{K}_{\mathrm{D}}$ is $4.9 \mu \mathrm{M}$ at $100 \mathrm{mM} \mathrm{NaCl}$ (Figure 5-A). Therefore we conclude the CTD is a DNA-binding domain; however, the specific structural interactions between the CTD and DNA are still unknown.

To examine if the CTD possessed discernible secondary structure, we characterized the solution-state structure of the CTD, using the SH3-CTD fusion protein, by solution-state NMR spectroscopy. Standard triple-resonance backbone assignment experiments were utilized to assign the whole SH3 domain and linker and 74\% of the CTD (all but the last 6 amino acids). The backbone resonances attributed to the CTD were used for CS-Rosetta structure determination ${ }^{40}$. The NMR data indicate that the CTD exists in a variety of conformations, which lack secondary structure (Figure 5-B). To corroborate the NMR results, we performed circular dichroism spectroscopy (CD) with synthetic CTD peptide. The resulting CD spectra were consistent with a disordered loop structure (Figure 5-C) in agreement with our NMR results. There is a possibility that the previously proposed helical CTD model ${ }^{6}$ was induced by binding to substrate. To test whether the isolated CTD changed its secondary structure as a function of substrate, we titrated dsDNA into CTD peptide and measured the resulting secondary structure by CD. The addition of substrate did not induce a conformational change in the CTD (Figure 5-C). Therefore, we conclude that the CTD is an intrinsically disordered polypeptide even in the presence of DNA substrate.

To further investigate the molecular mechanism of the CTD-DNA interaction, we performed molecular dynamics simulations 

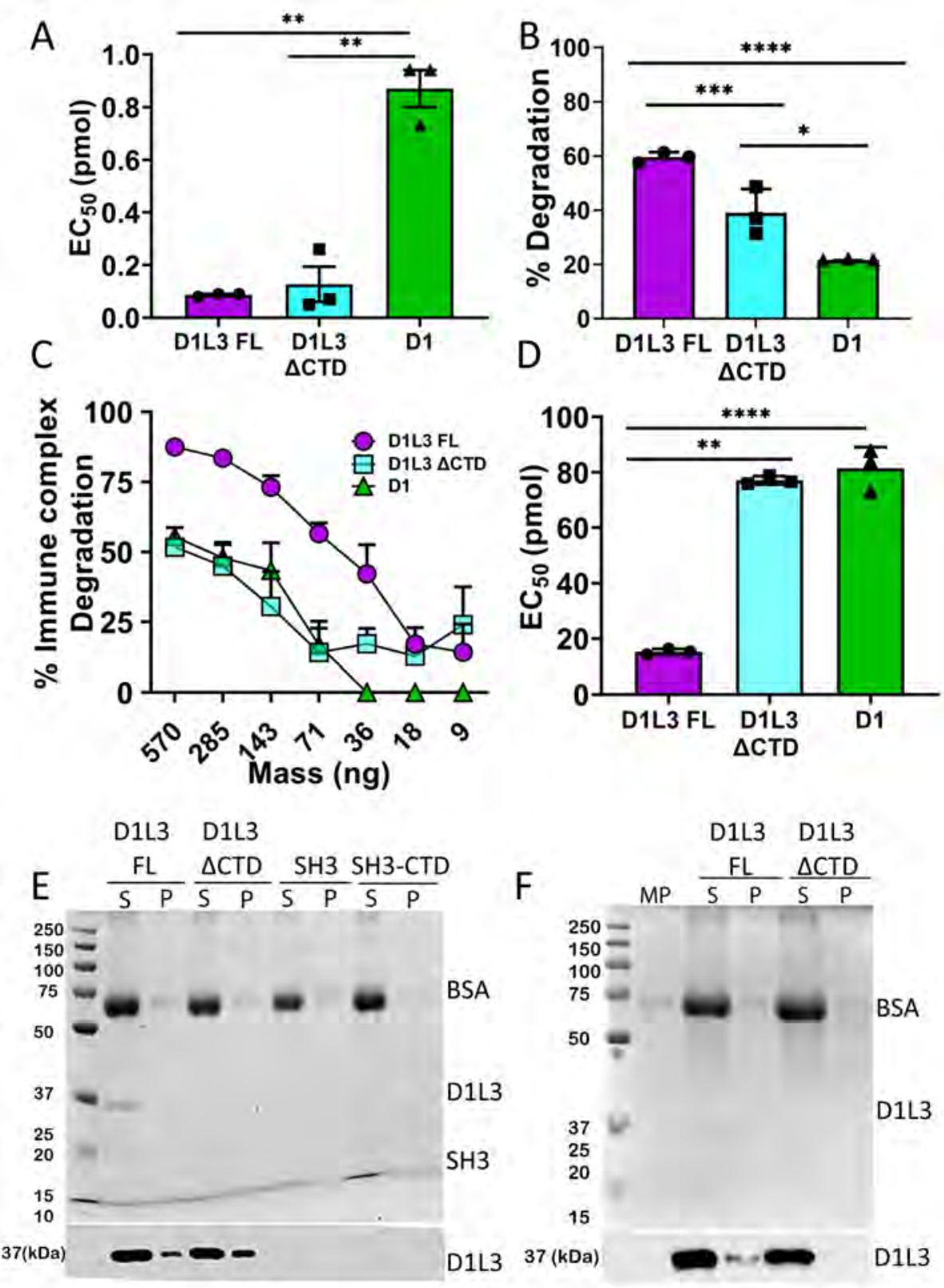

Figure 4. The CTD promotes complexed DNA degradation without lipid binding: (A) Dnase1 activities for full-length Dnase1L3 (D1L3 FL), Dnase1L3 $\Delta$ CTD (D1L3 $\Delta$ CTD) and Dnase1 (D1) were measured by mixing 200 ng of plasmid DNA with the reported range of Dnase concentrations for $30 \mathrm{~min}$ at $37^{\circ} \mathrm{C}$. (B) Dnase1L3-specific activity was measured using the barrier to transfection assay. HEK cells were transfected with $100 \mathrm{ng}$ of eGFP-N1 plasmid after plasmid-lipid complexes were incubated with $100 \mathrm{ng}$ of the indicated Dnase at $37^{\circ} \mathrm{C}$ for $30 \mathrm{~min}$. Transfection efficiency was measured by flow cytometry. (C, D) Dnase1L3-specific activity was monitored by measuring immune complex degradation. The indicated concentration of Dnase was incubated with chromatin-anti-dsDNA immune complexes, and the remaining anti-dsDNA antibody was measured. The percent immune complex degradation and $\mathrm{EC}_{50}$ were calculated as described in the methods. (E and F) The CTD does not promote lipid/DNA and microparticle/DNA binding: (E) Liposomes or (F) microparticles (MP) were incubated with wild type Dnase1L3 (D1L3FL), Dnase1L3 $\triangle$ CTD (D1L3 $\triangle$ CTD), SH3 or SH3-CTD and the supernatants (S) and pellets (P) were prepared in SDS sample buffer. Samples were resolved by SDS-PAGE followed by Coomassie staining (top) or transferred to nitrocellulose and probed with anti-Dnase1L3 (bottom). Graphs represent mean \pm SEM of three (A, C, D), or four (B) independent experiments. Each blot or Coomassie gel is a representative image from four independent experiments. ****p $<$ $0.0001, * * * \mathrm{p}<0.005, * * \mathrm{p}<0.01, * \mathrm{p}<0.05$. 

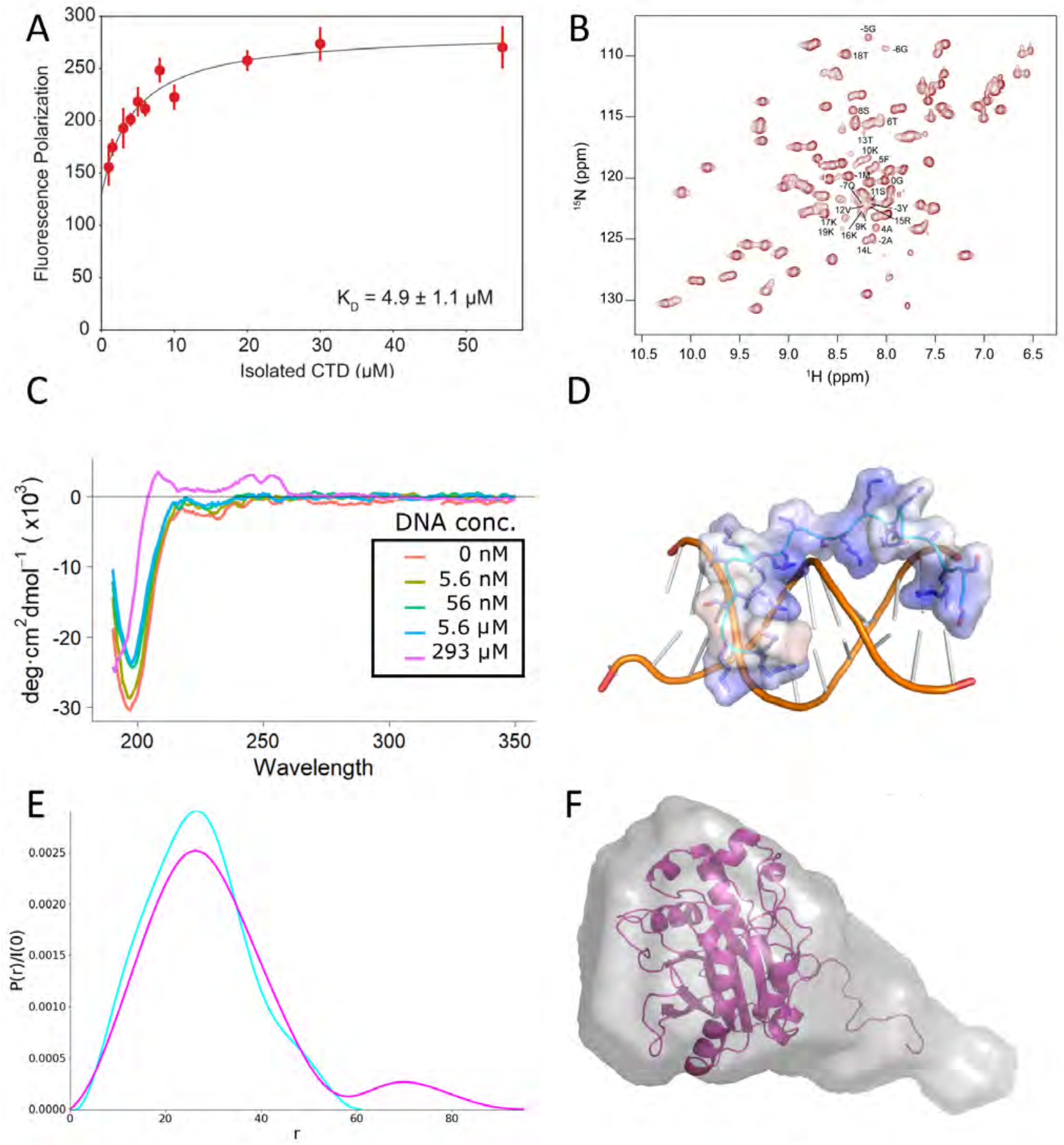

Figure 5. The CTD interacts with DNA. (A) Fluorescence Polarization of the CTD peptide with a fluorescently labeled 40-mer of dsDNA in $100 \mathrm{mM} \mathrm{NaCl}$. (B) NMR HSQC spectrum of SH3-CTD, which corresponds to the highly ordered and conserved SH3 domain and disordered CTD. (C) Far-UV Circular Dichroism of the CTD peptide with increasing concentration of substrate dsDNA. D) Snapshot of the CTD peptide interacting with DNA from MD simulation. E) Pair distribution function created using GNOM as implemented in BioXTAS RAW. The SAXS data indicates a population of scattering atoms $\sim 40 \AA$ from the centroid of Dnase1L3 that was identified as the CTD. F) SAXS bead model fit, calculated using MultiFOXS, of the combined Dnase1L3 + CTD model.

with a 16-mer of dsDNA and the NMR structure of the CTD equilibrated in an explicit solvent box. The MD simulations were run for a total of $1 \mu \mathrm{S}$ in NAMD using the CHARMM36m forcefield. Our MD results corroborate the proposed DNA 
localizing role of the CTD with an immediate attraction towards the dsDNA (Figure 5-D)(Supplementary Figure S9-A,B). The CTD was flexible during DNA binding and stabilized to two distinct conformations between molecular dynamics simulations (Supplementary Figure S9-C,D).The intrinsically disordered CTD remains disordered during interaction with DNA (Figure 5-C). In summary the intrinsically disordered C-terminal domain interacts with DNA to increase Dnase1L3's net affinity for its DNA substrate.

\section{The integration of the core enzyme and C-terminal domain structures}

The structure of full-length Dnase1L3 was solved using SAXS (Small Angle X-ray Scattering) to combine the high-resolution X-ray crystal and the CTD NMR structures. The solution-state SAXS profiles of full-length Dnase1L3 and Dnase1L3 $\Delta$ CTD were both collected to describe the CTD's contribution to the full-length enzyme. As expected, recombinant Dnase1L3 $\triangle \mathrm{CTD}$ is a globular protein in solution, with a $\mathrm{R}_{\mathrm{g}}$ of $21 \AA( \pm 0.4)$. The full-length Dnase1L3 maintains a similar $\mathrm{R}_{\mathrm{g}}(24.1 \AA \pm 2.5)$, but the pairwise distribution function indicates a population of scattering atoms roughly $44 \AA$ away from the centroid of the core domain (Figure 5-E). The distance between these two populations of scattering atoms correlates to an almost fully extended CTD separated from the core enzyme. Bead model reconstructions of full-length Dnase1L3 accounted for this extra density with an extended tail volume away from the globular core domain (Figure 5-F). MultiFOXS ${ }^{41}$ models generated from the indirect Fourier transform of full-length Dnase1L3 indicate a discrete solvent-exposed CTD (Figure 5-F). The pair distance distribution function obtained from solution-state SAXS analysis shows that full-length Dnase1L3 is a two-domain, pear-shaped molecule ${ }^{42}$ (Figure 5-F). Our results indicate that the CTD is an intrinsically disordered polypeptide that is not associated with the body of the core enzyme.

\section{Discussion}

Soluble serum nucleases such as Dnase1 and Dnase1L3 digest antigenic nucleic acids that are complexed within discarded membranes or with DNA-binding proteins. Given the complexed nature of the substrate DNA, it is less exposed and therefore less available for digestion by Dnase1, which can only digest a limited subset of complexed antigenic nucleic acids. Fortunately, the activity of Dnase1L3 is significantly broader ${ }^{6,8}$. The importance of Dnase1L3 in clearing antigenic DNA is highlighted by the human diseases that result from mutations within the DNASE1L3 gene. To understand Dnase1L3's broad range of nucleic acid degradation activity, we performed a structure-function analysis to resolve the features of Dnase1L3 responsible for the increased activity. We combined the high-resolution X-ray structure of the core domain with solution-state data of the intrinsically disordered C-terminal domain (CTD) to develop a structure of the complete Dnase1L3 molecule using Dnase1L3 expressed in E. coli.

Nucleases are notoriously difficult to overproduce in bacteria due to their DNA laddering activity on the exposed bacterial chromosome $^{18}$. We overcame this hurdle by expressing Dnase1L3 as an enzymatically inactive fusion protein. Dnase1L3 could be switched to an enzymatically active state through proteolytic cleavage of the MBP fusion partner and subsequent purification of the Dnase1L3 enzyme (Supplementary Figure S1-A). The MBP-Dnase1L3 fusion protein approach allowed us to take advantage of the folding enhancement from $\mathrm{MBP}^{43}$, in addition to the steric inhibition of Dnase1L3 provided by MBP during translation. We predict that this expression system can be utilized to produce other nucleases besides Dnase1L3.

The enzymatic activity of nucleases such as Dnase1 and Dnase1L3 are particularly important in diseases such as cystic fibrosis (CF). The viscous sputum that occurs within the lungs of CF patients contains DNA and actin ${ }^{44}$, both of which contribute to the abnormal visco-elasticity of pulmonary sputum ${ }^{45}$. Utilizing its role as a general nuclease, Dnase 1 can act as a mucolytic that digests the DNA component of sputum in the lower respiratory tract ${ }^{44}$. However, the excess of actin within the sputum abrogates the activity of native Dnase1. To address the actin limitation, Dnase1 has been reengineered to overcome actin inhibition (Pulmozyme®); however, in situ actin inhibition remains a therapeutic concern ${ }^{24}$. Dnase1L3 is naturally resistant to actin inhibition, in part, due to Phe-134 that disrupts the "central hydrophobic interface" with actin in Dnase $1^{12,25}$. A similar mechanism of actin resistance was proposed for Dnase1L2 ${ }^{46}$. Other determinants for actin resistance in Dnase1L3 include the disruption of stabilizing salt-bridged networks needed for actin binding to Dnase1, and the electrostatic repulsion of actin at the binding interface. Therefore, Dnase1L3 is naturally resistant to actin resistance because of both predicted and previously undescribed structural features.

The frequency of the R206C single nucleotide polymorphism (SNP) allele of DNASE1L3 is fairly common (1.7 to $7.7 \%$ ) in various populations ${ }^{26}$. In fact, R206C homozygosity is causally associated with rheumatoid arthritis ${ }^{47}$, systemic scleroderma ${ }^{48}$, and systemic lupus erythematosus (SLE) ${ }^{7}$. From a biochemical perspective, the R206C SNP displays decreased or absent ${ }^{26}$ enzyme activity and reduced enzyme secretion ${ }^{27}$. Previous literature explained the decreased enzyme activity in terms of increased mobility of the loop preceding Arg-206 (Dnase1L3 residues 193-196) that disrupts nucleic acid binding ${ }^{28}$. In addition, they conclude that only an interaction between Arg-206 and Glu-170 is relevant to the hypofunctional phenotype ${ }^{28}$. However, 
our crystallographic and molecular dynamics results reveal a more extensively salt-bridged network to Arg-206 associating with Asp-166, Glu-167 and Glu-170. Furthermore, we measure no significant change in loop mobility as a result of the R206C mutation. Our MD analysis indicates there is a general increase in the calculated free energy of folding as a result of the loss of the critical salt bridging interactions (Supplementary Figure S4, S5-A). Indeed, we find that the R206C mutation alters the calculated protein stability (Supplementary Figure S5-C) by $14 \mathrm{kcal} / \mathrm{mol}$. It therefore follows that errant protein folding would also affect extracellular secretion of the enzyme ${ }^{27}$ that results in a net hypofunctional Dnase1L3.

In our crystal structure and molecular dynamics simulations of Dnase1L3, Asn-191 coordinates $\mathrm{Mg}^{2+}$ 'IVa'. Indeed, the coordination of Asn-191 to $\mathrm{Mg}^{2+}$ ' IVa' is consistent with previous mutagenesis results ${ }^{19}$. Our analysis of the Dnase1L3 active site revealed a potential mechanism of His-155 regeneration via Arg-132, consistent with mutagenesis data and the proposed double divalent cation model ${ }^{11}$. The principal dissenting Dnase1 catalytic mechanism is the single-cation carboxylic acid model, which derives from the 4AWN crystal structure ${ }^{10}$. We show that Asp-189 in Dnase1L3 (homologous to Asp-168 in Dnase1) instead binds to the previously predicted second active site divalent cation, ' $\mathrm{IVa}^{30}$. The disagreement in $\mathrm{Mg}^{2+}$ coordination via Asp-168 (Asp-189 in Dnase1L3) can be explained by the more acidic pH of crystallization of $4 \mathrm{AWN}^{10}$. We provide the first structural evidence to support the double divalent cation mode ${ }^{11}$ for the Dnase 1 family. Our structural evidence clarifies the number of active site divalent cations and the role of several catalytic residues in the Dnase 1 family. Further investigation is needed to resolve the means of electrophilic attack and protonation of the 3'O on the scissile phosphate.

Aside from catalytic divalent cations, Dnase1L3 binds structural divalent cations differently than previously observed in Dnase1. The two missing divalent cation binding sites in Dnase1L3 have been described as structurally stabilizing binding sites in Dnase $1^{30}$. Furthermore, the disruption of the 'II' and 'III' binding sites in Dnase1 decreases the measured enzyme activity ${ }^{49}$. Nevertheless, in the absence of these stabilizing cations, the conformations of the catalytic residues in Dnase1L3 are similar to those observed in Dnase1. To address the perceived lack of stabilizing cations in Dnase1L3, we propose that the 'II' and 'III' sites observed in Dnase1 are unnecessary due in part to additional disulfide-mediated stabilization. The missing 'III' cation binding site is proposed to stabilize: the active site, the disulfide bridge, and nearby DNA-binding residues in Dnase ${ }^{49}$. In Dnase1L3, the absence of the Dnase1 'III' coordinating residue Asp-172 (Gly-193 in Dnase1L3) does not disrupt the disulfide located between Cys194-Cys231. The additional disulfide bond present in Dnase1L3 but not Dnase1, Cys24-Cys52, may allow for the reduced necessity of divalent cation-mediated stabilization.

Dnase1L3 displays an enhanced substrate diversity through its unique, highly basic C-terminal domain (CTD) ${ }^{5,6}$. Given the physiological role of Dnase1L3 and the exaggerated charge of its CTD, two binding targets were proposed: phospholipid membranes and DNA. Our biophysical characterization of Dnase1L3 reveals that the CTD is a physically separated, intrinsically disordered domain that binds dsDNA with $\mu \mathrm{M}$ affinity at physiological salt concentrations. The observation that the CTD of Dnase1L3 binds DNA with moderate affinity potentially facilitates an increased $\mathrm{K}_{\mathrm{off}}$ for rapid chromosomal laddering. Functionally, the intrinsically disordered CTD possesses the conformational versatility to bind complexed DNA in various forms, including nucleosomal, microparticle, and immune complexed DNA. We propose that the CTD is a conformationally flexible, disordered domain that enhances heterogeneous DNA binding and confers the unique activity attributed to Dnase1L3.

\section{Methods}

\section{Cloning and expression}

The genes for full-length human Dnase1L3 and the Dnase1L3 $\Delta$ CTD were sub-cloned into BamHI/XhoI sites in a Tobacco-Etch Protease (TEV) cleavable, his-tagged, maltose-binding protein (MBP) expression vector for E. coli expression. The expression plasmid was transformed into Rosetta-Gami®2 (DE3) competent cells (Novagen) to maintain disulfide bond formation. Single colonies were picked and grown to confluence in $100 \mathrm{ml}$ of LB media. $10 \mathrm{ml}$ of culture was inoculated into $1 \mathrm{~L}$ of Terrific Broth (TB) media, and grown to $\mathrm{OD}_{600}$ of 2.0. The culture was induced with $400 \mu \mathrm{M}$ IPTG and grown at $18{ }^{\circ} \mathrm{C}$ for 18 hours. Cells were harvested by centrifugation at $18,000 \mathrm{rpm}$ in an SS-40 rotor for 20 minutes. Cell pellets were frozen at $-80{ }^{\circ} \mathrm{C}$ until used for purification.

SH3-CTD was expressed in BL-21(DE3) E. coli cells in $3 \mathrm{~L}$ of Terrific Broth, induced with $400 \mu \mathrm{M}$ IPTG and grown at $18{ }^{\circ} \mathrm{C}$ for 18 hours. Cells were harvested by centrifugation, frozen in liquid $\mathrm{N}_{2}$ and stored at $-80{ }^{\circ} \mathrm{C}$ until ready for use.

\section{Purification of Full-length Dnase1L3, Dnase1L3 $\Delta$ CTD and SH3-CTD}

15-20 $\mathrm{g}$ of cells were resuspended in $160 \mathrm{ml}$ of lysis buffer (20 mM HEPES pH 7.4, $300 \mathrm{mM} \mathrm{NaCl,} 1 \mathrm{mM} \mathrm{CaCl}_{2}$ ). The cells were lysed using a microfluidizer and spun down at $19500 \mathrm{rpm}$ in a JA20 rotor for $50 \mathrm{~min}$. The supernatant was collected and run through a newly regenerated $\mathrm{Ni}^{2+} / \mathrm{NTA}$ column. The column was washed with lysis buffer that contained $30 \mathrm{mM}$ Imidazole. The protein was eluted with lysis buffer that contains $250 \mathrm{mM}$ imidazole, $5 \mathrm{mM}$ maltose. $0.1 \mathrm{mM}$ PMSF was added to the eluted sample. To remove the MBP fusion protein and to isolate the Dnase1L3, 2-3 mg of Tobacco Etch Virus protease were 
added to the sample and incubated at $4{ }^{\circ} \mathrm{C}$ overnight. An SDS gel was run to ensure the fusion protein was cut by TEV before moving on to the next purification step. The sample was diluted to $100 \mathrm{mM} \mathrm{NaCl}$ with Buffer A (20 mM Hepes pH 7.4, $1 \mathrm{mM}$ $\mathrm{CaCl}_{2}$ ). An SP-Sepharose (cation exchange) column was prepared and the sample was run through the column while collecting the flow-through (which contains the MBP). The column was then washed with Buffer A until the UV returned to the baseline before the sample was loaded onto the column. The protein was eluted with a gradient of $0-100 \% \mathrm{Buffer} \mathrm{B} \mathrm{(20} \mathrm{mM} \mathrm{Hepes} \mathrm{pH}$ 7.4, $1 \mathrm{M} \mathrm{NaCl}, 1 \mathrm{mM} \mathrm{CaCl}_{2}$ ) with a volume of at least three times the bed volume of the column. Purity was measured by SDS-PAGE. Finally, the sample was concentrated to run on an Superdex-75 gel filtration column equilibrated with Buffer C (20 mM HEPES pH 7.4, $400 \mathrm{mM} \mathrm{NaCl}, 1 \mathrm{mM} \mathrm{CaCl}_{2}$ ). The peak was then run on an SDS gel and imaged using a BioRad Stain-Free Gel imaging system.

To purify SH3-CTD, centrifuged bacteria were lysed by microfluidization and insoluble cell components were pelleted with $19,500 \mathrm{x}$ g centrifugation. SH3-CTD was separated from soluble lysate with 6-His Ni-NTA affinity chromatography. The high-affinity fraction containing SH3-CTD was eluted at $250 \mathrm{mM}$ Imidazole concentration. The elution was concentrated to 1 $\mathrm{ml}$ and injected into a $75 \mathrm{ml}$ Superdex-75 size exclusion column. The monodisperse fraction was exchanged into $1 \mathrm{M} \mathrm{NaCl}$ and incubated overnight with $1 \mathrm{mg}$ of TEV. The CTD was separated from the SH3 and TEV using Ni-NTA affinity chromatography, the CTD fraction came out in the FT. The CTD was buffer exchanged using $700 \mathrm{Da}$ cutoff filters into $100 \mathrm{mM} \mathrm{NaCl}$ and concentrated with lyophilization.

\section{Cell Culture}

HeLa cells (ATCC (Manassas, VA, USA), CCL-2) and HEK cells (ATCC CRL-1573) were cultured at $37{ }^{\circ} \mathrm{C}$ and $5 \% \mathrm{CO}_{2}$ in DMEM (Corning, Corning, NY, USA) supplemented with 10\% Equafetal bovine serum (Atlas, Fort Collins, CO), 1× L-glutamine (D10) and $1 \times$ penicillin/streptomycin. All cell lines were negative for mycoplasma.

\section{Antibodies}

The anti-Dnase1L3 rabbit polyclonal antibody was obtained from Abnova, and the anti-dsDNA monoclonal antibody was from the Developmental Studies Hybridoma Bank (DSHB, Iowa City, IA). Clone autoanti-dsDNA was deposited with the DSHB by Voss, E.W. (DSHB Hybridoma Product autoanti-dsDNA). HRP-conjugated secondary antibodies were from Jackson Immunoresearch (West Grove, Pennsylvania).

\section{Dnase1 Assay}

Dnase1 assays were performed as described ${ }^{50} .200 \mathrm{ng}$ plasmid DNA was incubated with varying concentrations of Dnase1L3 full length, Dnase1L3 $\Delta$ CTD or Dnase1 in Dnase assay buffer $\left(20 \mathrm{mM}\right.$ Tris, pH 7.4, $5 \mathrm{mM} \mathrm{MgCl}, 2 \mathrm{mM} \mathrm{CaCl}_{2}$ ) for $30 \mathrm{~min}$ at $37{ }^{\circ} \mathrm{C}$. The extent of DNA degradation was quantitated by measuring the integrated intensity of degraded and intact plasmid DNA from Gel Red-stained agarose gels using Photoshop Creative Suite (Adobe, San Jose, CA) and determining the percent degradation. The $\mathrm{EC}_{50}$ for Dnase1 activity was calculated from the dose-response curve using logistic regression.

\section{Barrier to Transfection}

HEK cells were plated one day prior to the assay at $5 \times 10^{5}$ cells per well in a 24 well plate. DNA-lipid complexes were prepared by incubating $100 \mathrm{ng}$ of eGFP-N1 plasmid (Takara Biosciences) with Lipofectamine 2000 for 20 min. DNA-lipid complexes were then incubated with $100 \mathrm{ng}$ each of full-length Dnase1L3, Dnase1L3 $\triangle \mathrm{CTD}$ or Dnase1 at $37^{\circ} \mathrm{C} 5 \% \mathrm{CO}_{2}$ for $30 \mathrm{minutes}$. HEK cells were then transfected with the control or the Dnase treated DNA-lipid complexes and incubated for $48 \mathrm{~h}$. The cells were supplemented with fresh D10 media after $24 \mathrm{~h}$. Cells were harvested, washed in FACS buffer ( $2 \%$ fetal calf serum, $0.05 \%$ $\mathrm{NaN}_{3}$ in 1x PBS), and analyzed on an Attune NxT flow cytometer. Transfection efficiency was $70 \%$ in control cells. Reduced transfection efficiency was calculated compared to control transfected cells.

\section{Immune Complex Degradation}

To measure immune complex degradation, a modified ELISA protocol was used. ELISA plates were precoated with 0.05 $\mathrm{mg} / \mathrm{mL}$ poly-L-lysine at room temp for $20 \mathrm{~min}$, washed with $1 \mathrm{x}$ nuclease-free water, and coated with $5 \mu \mathrm{g} / \mathrm{ml}$ calf thymus DNA (Sigma) overnight at $4{ }^{\circ} \mathrm{C}$. After washing $3 \mathrm{x}$ in PBS with $0.05 \%$ Tween (PBST) and blocking for $1 \mathrm{~h}$ at room temp with $1 \%$ BSA in PBST, $250 \mathrm{pg} / \mathrm{mL}$ anti-dsDNA antibody was added to all wells except the standard curve. The standard curve received 2-fold dilutions of anti-dsDNA antibody starting at $500 \mathrm{pg} / \mathrm{mL}$. After $1 \mathrm{~h}$, the plates were washed 3x in PBST, Dnase (diluted into $20 \mathrm{mM}$ HEPES, pH 7.4, $300 \mathrm{mM} \mathrm{NaCl}, 1 \mathrm{mM} \mathrm{CaCl}_{2}$ was added and plates incubated $37^{\circ} \mathrm{C}$ for $2 \mathrm{~h}$. Plates were washed $3 x$ in PBST, incubated with HRP conjugated goat anti-mouse IgG antibody (1:20,000), and developed using $10 \mathrm{mg} / \mathrm{ml} \mathrm{TMB}$ (Sigma), $3 \% \mathrm{H}_{2} \mathrm{O}_{2}$ in $100 \mathrm{mM}$ sodium acetate, $\mathrm{pH}$ 5.5. The reaction was stopped with $0.5 \mathrm{M} \mathrm{H}_{2} \mathrm{SO}_{4}$. $\mathrm{A}_{450}$ was measured and antibody concentration in each well calculated. The percentage of dsDNA antibody remaining in the well was calculated by comparison to control. Percentage immune complex degradation was 100 - \%remaining antibody. The $\mathrm{EC}_{50}$ for each $\mathrm{Dnase}$ was calculated using logistic regression. 


\section{Generation of Microparticles}

Microparticles from Hela cells were generated as previously described ${ }^{51}$. Cells were cultured overnight and then treated with 1 $\mathrm{mM}$ staurosporine (Sigma-Aldrich) for 8 hours in serum-free DMEM. Microparticles were harvested and debris removed by centrifugation for $5 \mathrm{~min}$ at $1,500 \mathrm{rpm}$. The supernatant was collected and centrifuged at 20,000 x g for 30 min to pellet the microparticles.

\section{Generation of Liposomes}

Synthetic Liposomes were prepared by mixing 20:20:10:50 mol percent of phosphatidylcholine (Avanti), phosphatidylethanolamine (Avanti), phosphatidylserine (Avanti) and cholesterol (Sigma). This lipid mixture was dried under $\mathrm{N}_{2}$ and resuspended to a final concentration of $4 \mathrm{mg} / \mathrm{mL}$ in liposome buffer (15 mM HEPES pH 7.2, $1 \mathrm{mM} \mathrm{Mg}\left(\mathrm{CH}_{3} \mathrm{COO}\right)_{2}$ and $50 \mathrm{mM}$ sorbitol). Liposomes were incubated at $37^{\circ} \mathrm{C}$ for $1 \mathrm{~h}$, freeze/thawed using dry ice $/ 37^{\circ} \mathrm{C}$ bath 5 times and stored at $-80^{\circ} \mathrm{C}$ until further use.

\section{Lipid and Microparticles Binding Assay}

Microparticles prepared from HeLa cells or $4 \mathrm{mg} / \mathrm{ml}$ liposomes were incubated with $10 \mathrm{mg} / \mathrm{mL}$ bovine serum albumin carrier protein and $6 \mu \mathrm{M}$ full length Dnase1L3, Dnase1L3 $\Delta \mathrm{CTD}$ or Dnase1 in 1x PBS with $\mathrm{Ca}^{2+}$ and $\mathrm{Mg}^{2+}$ on ice for $1 \mathrm{~h}$. Microparticles or liposomes were then centrifuged at $20,000 \times \mathrm{g} 4^{\circ} \mathrm{C}$ for $15 \mathrm{~min}$ and the supernatant saved for SDS-PAGE analysis. The pellet was washed with $1 \times$ PBS with $\mathrm{Ca}^{2+}$ and $\mathrm{Mg}^{2+}$ and centrifuged at $20,000 \times \mathrm{g} 4^{\circ} \mathrm{C}$ for 15 min. The resulting pellet was resuspended in SDS sample buffer. Samples were resolved on a $12.5 \%$ gel and either Coomassie-stained or transferred to a $0.45-\mu \mathrm{m}$ nitrocellulose membrane. Immunoblotting with anti-Dnase1L3 (1:1000) was followed by anti-rabbit IgG conjugated to HRP $(1: 10,000)$. Antibody staining was visualized on a FluorChemE (Protein Simple, San Jose, CA, USA) using enhanced chemiluminescence reagent [0.01\% $\mathrm{H}_{2} \mathrm{O}_{2}$ (Walmart, Bentonville, AR, USA), $0.2 \mathrm{mM}$ p-Coumaric acid (Sigma-Aldrich), $1.25 \mathrm{mM}$ Luminol (SigmaAldrich), and 0.1 M Tris, pH 8.4]. Immunoblots were analyzed using Photoshop Creative Suite 3.

\section{Fluorescence Polarization}

The dsDNA binding assays were performed using a FAM (carboxyfluorescein)-labeled 40-mer DNA (5'-FAM-GTGTTCGGACTCTGCCTCAAGACGGTAGTCAACGTGCTTG-3' and 5'-CAAGCACGTTGACTACCGTCTTGAGGCAGAGTCCGAACAC-3', IDT) ${ }^{52}$. dsDNA was made by annealing the FAM-labeled ssDNA to an unlabeled complementary strand at $95^{\circ} \mathrm{C}$. Fluorescence polarization (FP) experiments were performed in binding buffer (50 mM Hepes pH 7.5, $100 \mathrm{mM} \mathrm{NaCl}, 0.1 \mathrm{mM}$ EDTA, 1\%Glycerol, $1 \mathrm{mM}$ TCEP, $0.1 \%$ PEG $6000 \mathrm{w} / \mathrm{v}$ ), with $20 \mathrm{nM}$ dsDNA, and an increasing amount of isolated CTD for a final volume of $25 \mu \mathrm{L}$. Samples were incubated for 30 minutes at $37^{\circ} \mathrm{C}$ in a 384-well black plate (Fisher Scientific) before reading for FAM-FP in a Synergy Neo2 plate reader using the FP 485/530 filter. Three technical replicates were performed. The apparent dissociation constants were calculated by fitting the data to a modified version of the Hill equation ${ }^{53}$.

\section{Crystallization, Data Collection, Structure Solution and Refinement}

Purified Dnase1L3 $\triangle$ CTD was concentrated to $8 \mathrm{mg} / \mathrm{ml}$ and was screened with multiple sparse matrix crystallization kits. Initial crystal hits were expanded to improve crystal quality and yield more useful crystals for $\mathrm{X}$-ray analysis. The final crystallization condition was $19 \%$ PEG $8000,250 \mathrm{mM} \mathrm{MgCl} 2,100 \mathrm{mM}$ Tris, $\mathrm{pH} 8.5$ at $10^{\circ} \mathrm{C}$. The final Dnase $1 \mathrm{~L} 3 \Delta \mathrm{CTD}$ crystals grew to a final size of $\sim 0.5 \mathrm{~mm}$ in 60 days and were obelisk-shaped. The Dnase1L3 $\Delta$ CTD crystallized in the P1 space group with four molecules in the asymmetric unit (Table S1). The final X-ray dataset was collected at the SLAC beamline 14-1. The Dnase1L3 $\Delta$ CTD crystals diffracted to $1.9 \AA$.

The structure was solved with molecular replacement, as implemented in Phenix ${ }^{54}$, using the 1DNK structure as the target. $\mathrm{X}$-ray data to $2.2 \AA$ A was used for refinement. Electron density was fit using COOT $^{55}$ prior to additional rounds of structural refinement in Phenix. Each of the four molecules in the asymmetric unit was refined independently using Phenix ${ }^{54}$.

\section{Circular Dichroism}

A commercially synthesized peptide corresponding to the last 23 amino acids of Dnase1L3 (ie C-terminal domain (SSRAFTNSKKSVTLRKKTKSKRS)) (United BioSystem Inc, Herndon, VA, US) was resuspended from powder in milli-Q water to a stock concentration of $50 \mu \mathrm{M}$. The stock was diluted to $20 \mathrm{nM}$ in $5 \mathrm{mM} \mathrm{NaCl}$ with increasing concentrations of DNA from $0 \mathrm{nM}$ to $293 \mu \mathrm{M}$ of self-complementary oligomer DNA: GCGATCGCGCGATCGC. The circular dichroism data were collected on a Jasco J-815 CD spectrophotometer from $190 \mathrm{~nm}$ to $350 \mathrm{~nm}$. Buffer subtractions were the equivalent concentration of oligomer DNA in $5 \mathrm{mM} \mathrm{NaCl}$. The spectra were recorded in $\mathrm{CD}$ units of mdeg and then converted to molar ellipticity. The CTD was disordered throughout the measurements; however, the background subtraction became increasingly different from the Sample CD at high concentrations of DNA $(293 \mu \mathrm{M})$ likely corresponding to differences in the secondary structure of the DNA, even though the peptide remained disordered. The CD spectra of the CTD purified from the SH3-CTD construct was consistent with the synthesized peptide (data not shown). 


\section{NMR}

For NMR experiments, transformed Rosetta BL21 cells were grown in $2 \mathrm{x}$ minimal M9 media using ${ }^{15} \mathrm{NH}_{4} \mathrm{Cl}(1 \mathrm{~g} / \mathrm{L})$ and unlabeled or ${ }^{13} \mathrm{C}$ D-glucose $(3 \mathrm{~g} / \mathrm{L})$ as sole nitrogen and carbon sources, respectively. Protein expression was induced with $0.5 \mathrm{mM}$ isopropyl- $\beta$-d-thiogalactopyranoside for 18 hours at $25^{\circ} \mathrm{C}$. Bacterial cells were suspended in SH3 lysis buffer $(20$ $\mathrm{mM}$ HEPES pH 7.4, $300 \mathrm{mM} \mathrm{NaCl}$ ). Cell suspensions were lysed in a microfluidizer. Lysates were clarified by centrifugation at 19,500 rpm in a JA-20 rotor for $45 \mathrm{~min}$ at $4{ }^{\circ} \mathrm{C}$ and loaded on Ni-NTA resin pre-equilibrated with lysis buffer. SH3-CTD constructs were eluted in $250 \mathrm{mM}$ imidazole lysis buffer. The purification was followed by size exclusion chromatography in SH3 lysis buffer. SH3-CTD constructs were then buffer exchanged into $20 \mathrm{mM}$ Hepes $\mathrm{pH} 7.4,300 \mathrm{mM} \mathrm{NaCl}$, and $10 \% \mathrm{D}_{2} \mathrm{O}$ and concentrated to a desirable concentration $(0.7 \mathrm{mM})$ for NMR spectroscopy using a $5 \mathrm{kDa}$ molecular weight cutoff centrifugal concentrator (Millipore). All NMR experiments were performed on an Agilent $600 \mathrm{MHz}$ (14.1 T) DD2 NMR spectrometer equipped with a room temperature HCN z-axis gradient probe. NMR data were processed with NMRPipe/NMRDraw ${ }^{56}$ and analyzed with CCPN Analysis ${ }^{57}$. Backbone ${ }^{13} \mathrm{C} \alpha,{ }^{13} \mathrm{C} \beta,{ }^{13} \mathrm{C},{ }^{15} \mathrm{~N}$, and ${ }^{1} \mathrm{HN}$ resonance assignments of SH3-CTD were obtained from standard gradient-selected triple-resonance $\mathrm{HNCACB}, \mathrm{HN}(\mathrm{CO}) \mathrm{CACB}, \mathrm{HNCO}, \mathrm{HN}(\mathrm{CA}) \mathrm{CO}^{58}, \mathrm{HCCH}^{-}$ TOCSY $^{59}$, and NOESY HSQC ${ }^{60}$ experiments at $22^{\circ} \mathrm{C}$. Assignment data were collected with a random nonuniform sampling (NUS) scheme and reconstruction of NUS spectra was performed using Sparse Multidimensional Iterative Lineshape-Enhanced (SMILE) program ${ }^{61}$. The CTD and the linker between CTD and SH3 domains assignment were isolated by excluding the SH3 domain assigned residues using an assignment reference from the BMRB $(\text { code }=18054)^{39}$.

\section{SAXS Data Collection and Processing}

SAXS data were collected on a Xenocs BioXolver configured for SAXS/WAXS/GISAXS with a Genix 3D Cu HFVL source and a DECTRIS EIGER 1M detector. Purified full-length Dnase $1 \mathrm{~L} 3$ and $\triangle \mathrm{CTD}$ were concentrated to $4 \mathrm{mg} / \mathrm{ml}$. The enzyme was monodisperse, as determined by size exclusion chromatography, and was in $400 \mathrm{mM} \mathrm{NaCl}, 20 \mathrm{mM} \mathrm{HEPES}, 1 \mathrm{mM} \mathrm{CaCl}_{2}$. The filter concentrator flowthrough buffer was used for SAXS buffer subtraction. The proteins were irradiated five times for $300 \mathrm{sec}$ each and averaged before buffer subtraction with BioXtas-RAW. The Indirect Fourier Transform was performed using GNOM $^{62}$ with the limits based on the GNOM analysis of IFT quality. Bead models were constructed using Dammif $/ \mathrm{n}^{63}$. MultiFoXS ${ }^{41}$ was used in conjunction with the pairwise distribution function to refine the crystal structure of Dnase1L3 $\Delta$ CTD grafted with the CTD structure from NMR using comparative structure modeling ${ }^{64}$.

\section{Statistics}

Prism 5.0 (GraphPad, La Jolla, CA, USA) or Excel were used for statistical analysis. Data are represented as mean $\pm \mathrm{SEM}$ as indicated. The $\mathrm{EC}_{50}$ was calculated by logistic regression. Statistical significance was determined by one-way ANOVA or repeated measures ANOVA; $\mathrm{p}<0.05$ was statistically significant. Graphs were generated in R ggplot2, Excel and Photoshop.

\section{Molecular Dynamics}

All molecular dynamics input files were generated with CHARMM-GUI for NAMD with the CHARMM36m force field ${ }^{65}$. The high-resolution crystal structure of Dnase1L3 was used throughout for MD. The total charge of the system was neutralized by randomly substituting water molecules with $\mathrm{Mg}^{2+}$ and $\mathrm{Cl}^{-}$to obtain neutrality with $0.15 \mathrm{M}$ salt concentration. The TIP3 model for water was used throughout. A switching function was applied to the van der Waal's potential energy from 10 to $12 \AA$ to calculate nonbonded interactions. The Particle Mesh Ewald (PME) algorithm was used to calculate electrostatic interactions. Equilibration runs used the NVT ensemble at $300 \mathrm{~K}$. Energy minimization was performed for 10,000 steps to avoid any bad contacts generated while solvating the system. C- $\alpha$ restraints were generated from VMD. The simulations were analyzed using VMD. Production runs were performed on the HPCC Nocona cluster at Texas Tech University.

MD of CTD and DNA: The structure for the CTD, as determined by solution-state NMR, was isolated from the SH3 domain along with DNA based on the 1BNA 12-mer of dsDNA. The solution box was built with the CTD and a 12-mer (5'CGCGAATTCGCG-3') dsDNA molecule that was placed $\sim 30 \AA$ and $50 \AA$ from the CTD. The distances were required to minimize the bias of the simulation. The simulation was run for a total of one $\mu \mathrm{sec}$ with neutralizing $\mathrm{KCl}$. VMD and the Bio3D library for $\mathrm{R}$ were used to analyze the trajectories.

MD of Dnase1L3 $\triangle \mathrm{CTD}$ and DNA: DNA was added to the system based on a superposition of the 1DNK crystal structure of Dnase1 with DNA. Chain C of the Dnase1L3 high-resolution crystal structure was the starting structure for the MD runs with DNA. A $75 \AA$ x $75 \AA$ x $75 \AA$ water box with $150 \mathrm{mM} \mathrm{MgCl}_{2}$ (charge neutralized) was sized to the input Dnase1L3 molecule and was built using periodic boundary conditions. The simulated system contained $\sim 35,000$ solvent atoms, $\sim 69$ counter ions, and 4336 protein atoms. The crystallographic $\mathrm{Mg}^{2+}$ were retained. Three simulations were run for $1 \mu \mathrm{sec}$ each, starting from separate equilibration runs.

MD of Dnase1L3 R206C: The R206C mutation was generated with PyRosetta using Chain A of the crystal structure and 
Rosetta relaxed 3 times with the lowest energy score chosen prior to MD simulation. The solution box was built with $150 \mathrm{mM}$ $\mathrm{MgCl}_{2}$ and charge neutralization. The crystallographic $\mathrm{Mg}^{2+}$ were retained. A total of three production runs of $500 \mathrm{~ns}$ for Arg206 and Cys206 were generated for analysis. The last $100 \mathrm{~ns}$ (400ns to 500ns) were sufficient to reach a stable snapshot of each repeat. Ten frames, each $10 \mathrm{~ns}$ apart from the last $100 \mathrm{~ns}$ of the MD simulations were analyzed for free energy of folding using Rosetta, with the lowest energy score, REF-2015, after three full Rosetta relax cycles compared between Arg206 and Cys206. A one-way ANOVA, (alpha=0.05) was performed to highlight the difference between the single comparison of significance, Arg-206 or Cys206 on free energy of folding. The average computed free energy of folding from 10 timestamps across 3 simulations, for both WT and R206C Dnase1L3, was measured as -664.99 $(n=30)$ for WT (Arg206) and -650.96 $(\mathrm{n}=30)$ for R206C. The standard deviation for WT free energy of folding was 13.633, and 15.642 for R206C. The difference in folding was determined to be $14.03 \pm 3.79$ (s.d.), p-value $=0.000476$.

\section{Iso-electric point calculations}

Iso-electric calculations were computed using $\mathrm{H}++^{66-68}$. For the actin-binding loop following the actin-binding helix in Dnase $1^{22}$, and the homologous loop in Dnase1L3, the regions of interest were isolated in Pymol and processed using the H++ web server at a $\mathrm{pH}$ of 7 , with a salinity of $0.15 \mathrm{M}$, internal dielectric of 0.1 , and external dielectric of 80 .

\section{pKa of Active Site Residues:}

The computational determination of acid dissociation constant was done with a local installation of DelPhi-Pka ${ }^{37}$. All four Dnase1L3 molecules in the asymmetric unit and each available Dnase1 structure ${ }^{10,22,32-36}$, were processed for pKa calculation in DelPhi-Pka after being cleaned of any hetero-atoms.

\section{References}

1. Tsokos, G. C. Systemic lupus erythematosus. New Engl. J. Medicine 365, 2110-2121, DOI: 10.1056/nejmra1100359 (2011).

2. Özçakar, Z. B. et al. Dnase1L3 mutations in hypocomplementemic urticarial vasculitis syndrome. Arthritis \& Rheum. $\mathbf{6 5}$, 2183-2189, DOI: 10.1002/art.38010 (2013).

3. Zhong, X.-Y. et al. Increased concentrations of antibody-bound circulatory cell-free DNA in rheumatoid arthritis. Clin. Chem. 53, 1609-1614, DOI: 10.1373/clinchem.2006.084509 (2007).

4. Jiménez-Alcázar, M. et al. Host DNases prevent vascular occlusion by neutrophil extracellular traps. Science 358, 1202-1206, DOI: 10.1126/science.aam8897 (2017).

5. Keyel, P. A. Dnases in health and disease. Dev. Biol. 429, 1-11, DOI: 10.1016/j.ydbio.2017.06.028 (2017).

6. Sisirak, V. et al. Digestion of chromatin in apoptotic cell microparticles prevents autoimmunity. Cell 166, 88-101, DOI: 10.1016/j.cell.2016.05.034 (2016).

7. Al-Mayouf, S. M. et al. Loss-of-function variant in Dnase1L3 causes a familial form of systemic lupus erythematosus. Nat. Genet. 43, 1186-1188, DOI: 10.1038/ng.975 (2011).

8. Hartl, J. et al. Autoantibody-mediated impairment of Dnase1L3 activity in sporadic systemic lupus erythematosus. J. Exp. Medicine 218, DOI: 10.1084/jem.20201138 (2021).

9. Engavale, M. et al. Dnase1 family in autoimmunity. Encyclopedia 1, 527-541, DOI: 10.3390/encyclopedia1030044 (2021).

10. Parsiegla, G., Noguere, C., Santell, L., Lazarus, R. A. \& Bourne, Y. The structure of human Dnase I bound to magnesium and phosphate ions points to a catalytic mechanism common to members of the Dnase I-like superfamily. Biochemistry 51, 10250-10258, DOI: 10.1021/bi300873f (2012).

11. Jones, S. J., Worrall, A. F. \& Connolly, B. A. Site-directed mutagenesis of the catalytic residues of bovine pancreatic deoxyribonuclease I. J. Mol. Biol. 264, 1154-1163, DOI: 10.1006/jmbi.1996.0703 (1996).

12. Lazarus, R. A. \& Wagener $\dagger$, J. S. Recombinant human deoxyribonuclease i. In Pharmaceutical Biotechnology, 471-488, DOI: 10.1007/978-3-030-00710-2_22 (Springer International Publishing, 2019).

13. Ribeiro, A. J. M. et al. Mechanism and catalytic site atlas (m-CSA): a database of enzyme reaction mechanisms and active sites. Nucleic Acids Res. 46, D618-D623, DOI: 10.1093/nar/gkx1012 (2017). 
14. Napirei, M., Ludwig, S., Mezrhab, J., Klöckl, T. \& Mannherz, H. G. Murine serum nucleases - contrasting effects of plasmin and heparin on the activities of Dnase1 and Dnase1-like-3 (Dnase1L3). FEBS J. 276, 1059-1073, DOI: 10.1111/j.1742-4658.2008.06849.x (2009).

15. Soni, C. \& Reizis, B. Self-DNA at the epicenter of SLE: Immunogenic forms, regulation, and effects. Front. Immunol. 10, DOI: 10.3389/fimmu.2019.01601 (2019).

16. Wilber, A., Lu, M. \& Schneider, M. C. Deoxyribonuclease 1-like 3 is an inducible macrophage barrier to liposomal transfection. Mol. Ther. 6, 35-42, DOI: 10.1006/mthe.2002.0625 (2002).

17. Jumper, J. et al. Highly accurate protein structure prediction with AlphaFold. Nature DOI: 10.1038/s41586-021-03819-2 (2021).

18. Doherty, A. J., Connolly, B. A. \& Worrall, A. F. Overproduction of the toxic protein, bovine pancreatic DNasel, in escherichia coli using a tightly controlled T7-promoter-based vector. Gene 136, 337-340, DOI: 10.1016/0378-1119(93) 90491-k (1993).

19. Pan, C. Q., Uumer, J. S., Herzka, A. \& Lazarus, R. A. Mutational analysis of human DNase I at the DNA binding interface: Implications for DNA recognition, catalysis, and metal ion dependence. Protein Sci. 7, 628-636, DOI: 10.1002/pro.5560070312 (1998).

20. Lam, C. et al. Taming hyperactive hDNase I: Stable inducible expression of a hyperactive salt- and actin-resistant variant of human deoxyribonuclease I in CHO cells. Biotechnol. Prog. 33, 523-533, DOI: 10.1002/btpr.2439 (2017).

21. Fujihara, J. et al. Two N-Linked Glycosylation Sites (Asn18 and Asn106) Are Both Required for Full Enzymatic Activity, Thermal Stability, and Resistance to Proteolysis in Mammalian Deoxyribonuclease I. Biosci. Biotechnol. Biochem. 72, 3197-3205, DOI: 10.1271/bbb.80376 (2008).

22. Kabsch, W., Mannherz, H. G., Suck, D., Pai, E. F. \& Holmes, K. C. Atomic structure of the actin: DNase I complex. Nature 347, 37-44, DOI: 10.1038/347037a0 (1990).

23. Pan, C. Q. et al. Improved potency of hyperactive and actin-resistant human Dnase I variants for treatment of cystic fibrosis and systemic lupus erythematosus. J. Biol. Chem. 273, 18374-18381, DOI: 10.1074/jbc.273.29.18374 (1998).

24. Bucki, R. et al. Enhancement of pulmozyme activity in purulent sputum by combination with poly-aspartic acid or gelsolin. J. Cyst. Fibros. 14, 587-593, DOI: 10.1016/j.jcf.2015.02.001 (2015).

25. Ulmer, J. S. et al. Engineering actin-resistant human DNase I for treatment of cystic fibrosis. Proc. Natl. Acad. Sci. 93, 8225-8229, DOI: 10.1073/pnas.93.16.8225 (1996).

26. Ueki, M. et al. Caucasian-specific allele in non-synonymous single nucleotide polymorphisms of the gene encoding deoxyribonuclease 1-like 3, potentially relevant to autoimmunity, produces an inactive enzyme. Clin. Chimica Acta 407, 20-24, DOI: 10.1016/j.cca.2009.06.022 (2009).

27. Coke, L. N. et al. Arg206Cys substitution in Dnase1L3 causes a defect in Dnase1L3 protein secretion that confers risk of systemic lupus erythematosus. Annals Rheum. Dis. 80, 782-787, DOI: 10.1136/annrheumdis-2020-218810 (2021).

28. Zervou, M. et al. Association of the Dnase1L3 rs35677470 polymorphism with systemic lupus erythematosus, rheumatoid arthritis and systemic sclerosis: Structural biological insights. Mol. Medicine Reports 22, 4492-4498, DOI: 10.3892/mmr. 2020.11547 (2020).

29. Alford, R. F. et al. The rosetta all-atom energy function for macromolecular modeling and design. J. Chem. Theory Comput. 13, 3031-3048, DOI: 10.1021/acs.jctc.7b00125 (2017).

30. Guéroult, M., Picot, D., Abi-Ghanem, J., Hartmann, B. \& Baaden, M. How cations can assist DNase1 in DNA binding and hydrolysis. PLoS Comput. Biol. 6, e1001000, DOI: 10.1371/journal.pcbi.1001000 (2010).

31. Worrall, A. F. \& Connolly, B. A. The chemical synthesis of a gene coding for bovine pancreatic DNase I and its cloning and expression in Escherichia coli. J Biol Chem 265, 21889-21895 (1990).

32. Weston, S., Lahm, A. \& Suck, D. X-ray structure of the Dnase I-d(GGTATACC) 2 complex at 2 A resolution. J. Mol. Biol. 226, 1237-1256, DOI: 10.1016/0022-2836(92)91064-v (1992).

33. Chereau, D. et al. Actin-bound structures of Wiskott-Aldrich syndrome protein (WASP)-homology domain 2 and the implications for filament assembly. Proc. Natl. Acad. Sci. 102, 16644-16649, DOI: 10.1073/pnas.0507021102 (2005). 
34. Lahm, A. \& Suck, D. DNase I-induced DNA conformation. J. Mol. Biol. 222, 645-667, DOI: 10.1016/0022-2836(91) 90502-w (1991).

35. Kudryashov, D. S. et al. Connecting actin monomers by iso-peptide bond is a toxicity mechanism of the Vibrio cholerae MARTX toxin. Proc. Natl. Acad. Sci. 105, 18537-18542, DOI: 10.1073/pnas.0808082105 (2008).

36. Oefner, C. \& Suck, D. Crystallographic refinement and structure of DNase1 at 2 A resolution. J. Mol. Biol. 192, 605-632, DOI: $10.1016 / 0022-2836(86) 90280-9$ (1986).

37. Pahari, S., Sun, L., Basu, S. \& Alexov, E. DelPhiPKa: Including salt in the calculations and enabling polar residues to titrate. Proteins: Struct. Funct. Bioinforma. 86, 1277-1283, DOI: 10.1002/prot.25608 (2018).

38. Chen, W.-J. et al. Probing the catalytic mechanism of bovine pancreatic deoxyribonuclease I by chemical rescue. Biochem. Biophys. Res. Commun. 352, 689-696, DOI: 10.1016/j.bbrc.2006.11.078 (2007).

39. Stollar, E. J., Lin, H., Davidson, A. R. \& Forman-Kay, J. D. Differential dynamic engagement within 24 SH3 domain: Peptide complexes revealed by co-linear chemical shift perturbation analysis. PLoS ONE 7, e51282, DOI: 10.1371/journal. pone.0051282 (2012).

40. Shen, Y. et al. Consistent blind protein structure generation from NMR chemical shift data. Proc. Natl. Acad. Sci. 105, 4685-4690, DOI: 10.1073/pnas.0800256105 (2008).

41. Schneidman-Duhovny, D., Hammel, M., Tainer, J. A. \& Sali, A. FoXS, FoXSDock and MultiFoXS: Single-state and multistate structural modeling of proteins and their complexes based on SAXS profiles. Nucleic Acids Res. 44, W424-W429, DOI: 10.1093/nar/gkw389 (2016).

42. Svergun, D. I. \& Koch, M. H. J. Small-angle scattering studies of biological macromolecules in solution. Reports on Prog. Phys. 66, 1735-1782, DOI: 10.1088/0034-4885/66/10/r05 (2003).

43. Raran-Kurussi, S. \& Waugh, D. S. The ability to enhance the solubility of its fusion partners is an intrinsic property of maltose-binding protein but their folding is either spontaneous or chaperone-mediated. PLOS ONE 7, e49589, DOI: 10.1371/journal.pone.0049589 (2012).

44. Sheils, C. A. et al. Actin filaments mediate DNA fiber formation in chronic inflammatory airway disease. Am J Pathol 148, 919-927 (1996).

45. Bakker, E. M. et al. Improved treatment response to dornase alfa in cystic fibrosis patients using controlled inhalation. Eur. Respir. J. 38, 1328-1335, DOI: 10.1183/09031936.00006211 (2011).

46. Delfino, D. et al. Actin-resistant Dnase1L2 as a potential therapeutics for CF lung disease. Biomolecules 11, 410, DOI: 10.3390/biom11030410 (2021).

47. Westra, H.-J. et al. Fine-mapping and functional studies highlight potential causal variants for rheumatoid arthritis and type 1 diabetes. Nat. Genet. 50, 1366-1374, DOI: 10.1038/s41588-018-0216-7 (2018).

48. Mayes, M. D. et al. Immunochip analysis identifies multiple susceptibility loci for systemic sclerosis. The Am. J. Hum. Genet. 94, 47-61, DOI: 10.1016/j.ajhg.2013.12.002 (2014).

49. Chen, W.-J. Biological functions of the disulfides in bovine pancreatic deoxyribonuclease. Protein Sci. 13, 875-883, DOI: 10.1110/ps.03438204 (2004).

50. Shi, G., Abbott, K. N., Wu, W., Salter, R. D. \& Keyel, P. A. Dnase1L3 regulates inflammasome-dependent cytokine secretion. Front. Immunol. 8, DOI: 10.3389/fimmu.2017.00522 (2017).

51. Ullal, A. J. et al. Microparticles as antigenic targets of antibodies to DNA and nucleosomes in systemic lupus erythematosus. J. Autoimmun. 36, 173-180, DOI: 10.1016/j.jaut.2011.02.001 (2011).

52. Majka, J., Alford, B., Ausio, J., Finn, R. M. \& McMurray, C. T. ATP hydrolysis by RAD50 protein switches MRE11 enzyme from endonuclease to exonuclease. J. Biol. Chem. 287, 2328-2341, DOI: 10.1074/jbc.m111.307041 (2012).

53. Pagano, J. M., Clingman, C. C. \& Ryder, S. P. Quantitative approaches to monitor protein-nucleic acid interactions using fluorescent probes. RNA 17, 14-20, DOI: 10.1261/rna.2428111 (2010).

54. Afonine, P. V. et al. Towards automated crystallographic structure refinement with phenix.refine. Acta Crystallogr. Sect. D Biol. Crystallogr. 68, 352-367, DOI: 10.1107/s0907444912001308 (2012). 
55. Emsley, P. \& Cowtan, K. Coot: model-building tools for molecular graphics. Acta Crystallogr. Sect. D Biol. Crystallogr. 60, 2126-2132, DOI: 10.1107/s0907444904019158 (2004).

56. Delaglio, F. et al. NMRPipe: A multidimensional spectral processing system based on UNIX pipes. J. Biomol. NMR 6, DOI: $10.1007 / \mathrm{bf00197809} \mathrm{(1995).}$

57. Vranken, W. F. et al. The CCPN data model for NMR spectroscopy: Development of a software pipeline. Proteins: Struct. Funct. Bioinforma. 59, 687-696, DOI: 10.1002/prot.20449 (2005).

58. Venters, R. A., II, B. T. F., Fierke, C. A. \& Spicer, L. D. Characterizing the use of perdeuteration in NMR studies of large proteins: ${ }^{13} \mathrm{C},{ }^{15} \mathrm{~N}$ and ${ }^{1} \mathrm{H}$ assignments of human carbonic anhydrase II. J. Mol. Biol. 264, 1101-1116, DOI: 10.1006/jmbi.1996.0699 (1996).

59. Bax, A., Clore, G. \& Gronenborn, A. M. ${ }^{1} \mathrm{H}-{ }^{1} \mathrm{H}$ correlation via isotropic mixing of ${ }^{13} \mathrm{C}$ magnetization, a new threedimensional approach for assigning ${ }^{1} \mathrm{H}$ and ${ }^{13} \mathrm{C}$ spectra of ${ }^{13} \mathrm{C}$-enriched proteins. J. Magn. Reson. (1969) 88, 425-431, DOI: 10.1016/0022-2364(90)90202-k (1990).

60. Shimotakahara, S. et al. NMR structural analysis of an analog of an intermediate formed in the rate-determining step of one pathway in the oxidative folding of bovine pancreatic Ribonuclease A: Automated analysis of ${ }^{1} \mathrm{H},{ }^{13} \mathrm{C}$, and ${ }^{15} \mathrm{~N}$ resonance assignments for wild-type and [C65S, C72S] mutant forms. Biochemistry 36, 6915-6929, DOI: 10.1021/bi963024k (1997).

61. Ying, J., Delaglio, F., Torchia, D. A. \& Bax, A. Sparse multidimensional iterative lineshape-enhanced (SMILE) reconstruction of both non-uniformly sampled and conventional NMR data. J. Biomol. NMR 68, 101-118, DOI: 10.1007/s10858-016-0072-7 (2016).

62. Svergun, D. I. Determination of the regularization parameter in indirect-transform methods using perceptual criteria. $J$. Appl. Crystallogr. 25, 495-503, DOI: 10.1107/s0021889892001663 (1992).

63. Manalastas-Cantos, K. et al. ATSAS 3.0: expanded functionality and new tools for small-angle scattering data analysis. $J$. Appl. Crystallogr. 54, 343-355, DOI: 10.1107/s1600576720013412 (2021).

64. Webb, B. \& Sali, A. Comparative protein structure modeling using MODELLER. Curr. Protoc. Bioinforma. 54, DOI: 10.1002/cpbi.3 (2016).

65. Jo, S., Kim, T., Iyer, V. G. \& Im, W. CHARMM-GUI: A web-based graphical user interface for CHARMM. J. Comput. Chem. 29, 1859-1865, DOI: 10.1002/jcc.20945 (2008).

66. Anandakrishnan, R., Aguilar, B. \& Onufriev, A. V. H++3.0: automating pK prediction and the preparation of biomolecular structures for atomistic molecular modeling and simulations. Nucleic Acids Res. 40, W537-W541, DOI: 10.1093/nar/ gks375 (2012).

67. Myers, J., Grothaus, G., Narayanan, S. \& Onufriev, A. A simple clustering algorithm can be accurate enough for use in calculations of pKs in macromolecules. Proteins: Struct. Funct. Bioinforma. 63, 928-938, DOI: 10.1002/prot.20922 (2006).

68. Gordon, J. C. et al. H++: a server for estimating pKas and adding missing hydrogens to macromolecules. Nucleic Acids Res. 33, W368-W371, DOI: 10.1093/nar/gki464 (2005).

69. Sutoh, K. Actin-actin and actin-deoxyribonuclease i contact sites in the actin sequence. Biochemistry 23, 1942-1946, DOI: 10.1021/bi00304a009 (1984).

70. Anderson, D. E., Becktel, W. J. \& Dahlquist, F. W. pH-induced denaturation of proteins: a single salt bridge contributes 3-5 $\mathrm{kcal} / \mathrm{mol}$ to the free energy of folding of t4 lysozyme. Biochemistry 29, 2403-2408, DOI: 10.1021/bi00461a025 (1990).

\section{Acknowledgements}

This publication was supported by the Lupus Research Alliance, The $\underline{\mathrm{CH}}$ Foundation, the Lubbock Lupus Group, and the National Institute of General Medical Sciences (1R35GM128906; MPL) and a seed grant provided by the TTUHSC Center for Membrane Protein Research. Use of the Stanford Synchrotron Radiation Lightsource, SLAC National Accelerator Laboratory, is supported by the U.S. Department of Energy, Office of Science, Office of Basic Energy Sciences under Contract No. DE-AC0276SF00515. The SSRL Structural Molecular Biology Program is supported by the DOE Office of Biological and Environmental Research, and by the National Institutes of Health, National Institute of General Medical Sciences (P30GM133894). The 
contents of this publication are solely the responsibility of the authors and do not necessarily represent the official views of funding agencies, including the LRA, NIGMS or NIH.

The authors acknowledge the Texas Tech University College of Arts and Sciences Microscopy for flow cytometry and the High-Performance Computing Center (HPCC) at Texas Tech University for providing computational resources that have contributed to the research results reported within this paper. URL: http://www.hpcc.ttu.edu

The authors also thank Dr. Faraz Harsini for help with the initial Dnase1L3 purification.

\section{Author contributions statement}

PAK, RBS, and MPL conceived the experiment(s), JJM, ME, EM, JV, BM conducted the experiment(s), RBS, PAK, and MPL analyzed the results. All authors reviewed the manuscript.

\section{Additional information}

All crystallographic coordinates and structure factors have been deposited in the PDB under the accession code 7KIU. 


\section{Supplementary Material}

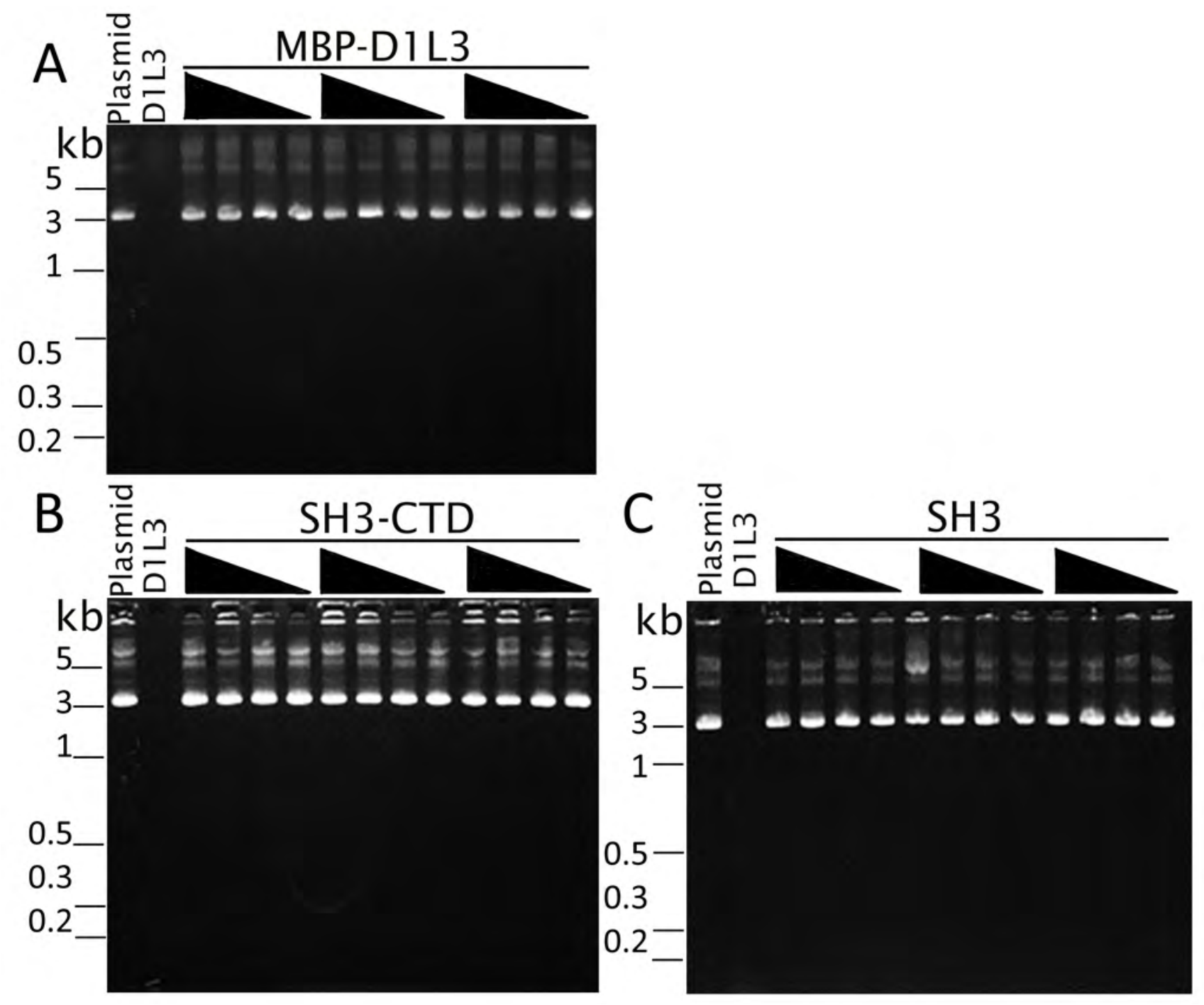

Figure S1. Dnase1 activity requires the core Dnase1L3 enzyme: (A) Dnase1 activity of MBP fusion protein Dnase1L3 (D1L3 MBP), (B) SH3-CTD fusion or (C) SH3 domain alone was measured by mixing 200 ng of plasmid DNA with a range of concentrations for $30 \mathrm{~min}$ at $37^{\circ} \mathrm{C}$ and analyzing on an agarose gel. The first lane in each sample is the DNA-only negative control. The second lane is full-length Dnase1L3 as a positive control. 


\begin{tabular}{|c|c|}
\hline & Dnase1L3 $\Delta$ CTD (S283X) \\
\hline Wavelength $(\AA)$ & 1.12709 \\
\hline Resolution Range $(\AA)$ & $32.06-2.22(2.299-2.22)$ \\
\hline Space Group & $\mathrm{P} 1$ \\
\hline Unit Cell $(\AA)$ & \\
\hline a, b, c $(\AA)$ & $52.313,72.671,73.169$ \\
\hline$\alpha, \beta, \gamma\left(^{\circ}\right)$ & $85.394,80.558,88.392$ \\
\hline Total Reflections & $454844(18459)$ \\
\hline Unique Reflections & 49718 (4702) \\
\hline Multiplicity & $9.1(3.9)$ \\
\hline Completeness (\%) & $93.95(79.91)$ \\
\hline Mean I/ $\sigma(\mathrm{I})$ & $8.59(1.01)$ \\
\hline Wilson B-factor & 51.22 \\
\hline $\mathrm{R}_{\text {merge }}(\%)$ & $14.51(128)$ \\
\hline $\mathrm{CC}_{1 / 2}(\%)$ & $99.8(52.6)$ \\
\hline Reflections used in Refinement & $49177(4189)$ \\
\hline Reflections used for R-free & $2218(189)$ \\
\hline $\mathrm{R}_{\mathrm{work}}(\%)$ & $19.06(50.29)$ \\
\hline $\mathrm{R}_{\text {free }}(\%)$ & $23.04(52.09)$ \\
\hline Number of non-hydrogen atoms & 8915 \\
\hline macromolecules & 8729 \\
\hline ligands & 12 \\
\hline Solvent & 174 \\
\hline Protein residues & 1052 \\
\hline RMS (bonds, Å) & 0.015 \\
\hline RMS (angles, ${ }^{\circ}$ ) & 1.74 \\
\hline Ramachandran favored (\%) & 95.40 \\
\hline Ramachandran allowed (\%) & 4.60 \\
\hline Ramachandran outliers (\%) & 0.00 \\
\hline Rotamer outliers (\%) & 4.27 \\
\hline Clashscore & 4.08 \\
\hline Average B-factor $\left(\AA^{2}\right)$ & 64.95 \\
\hline macromolecules $\left(\AA^{2}\right)$ & 65.19 \\
\hline ligands $\left(\AA^{2}\right)$ & 55.39 \\
\hline solvent $\left(\AA^{2}\right)$ & 53.89 \\
\hline
\end{tabular}

Table S1. Crystallographic Data and Refinement Summary. 
bioRxiv preprint doi: https://doi.org/10.1101/2022.01.19.476959; this version posted January 20, 2022. The copyright holder for this preprint (which was not certified by peer review) is the author/funder. All rights reserved. No reuse allowed without permission.
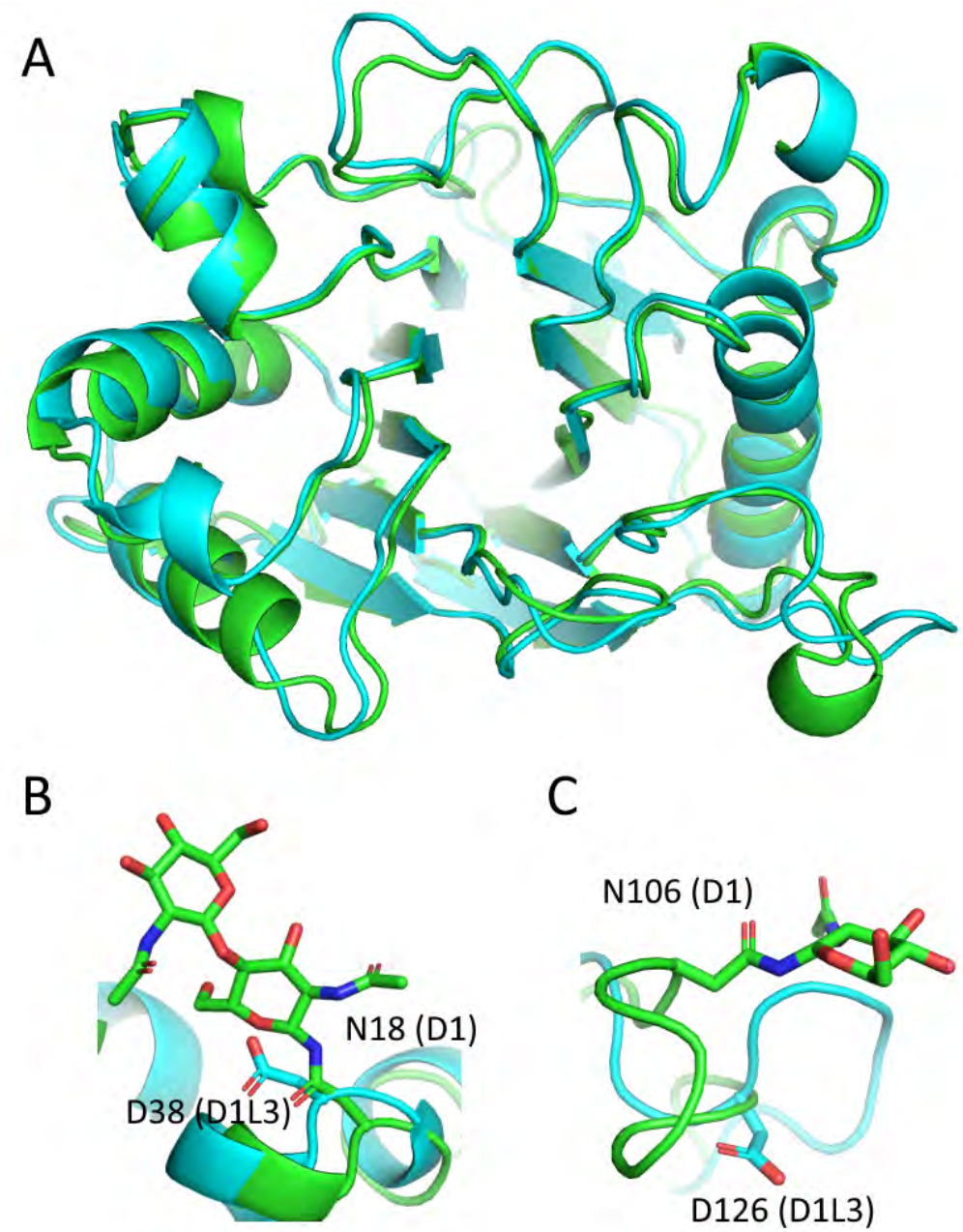

C

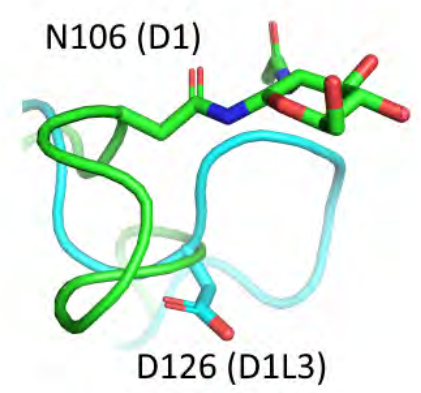

Figure S2. (A) The crystal structure of Chain C of Dnase1L3 $\Delta$ CTD (PDB:7KIU) in blue aligned to Dnase1 (PDB:4AWN) in green using $\mathrm{C}-\alpha$ alignment with an RMSD $=1.76 \AA$. (B) The homologous residue to the N-linked glycosylation site (Asn-18) in Dnase1 is Asp-38 in Dnase1L3. (C) The Asn-106 N-linked glycosylation site in Dnase1 corresponds to Asp 126 in Dnase1L3. 


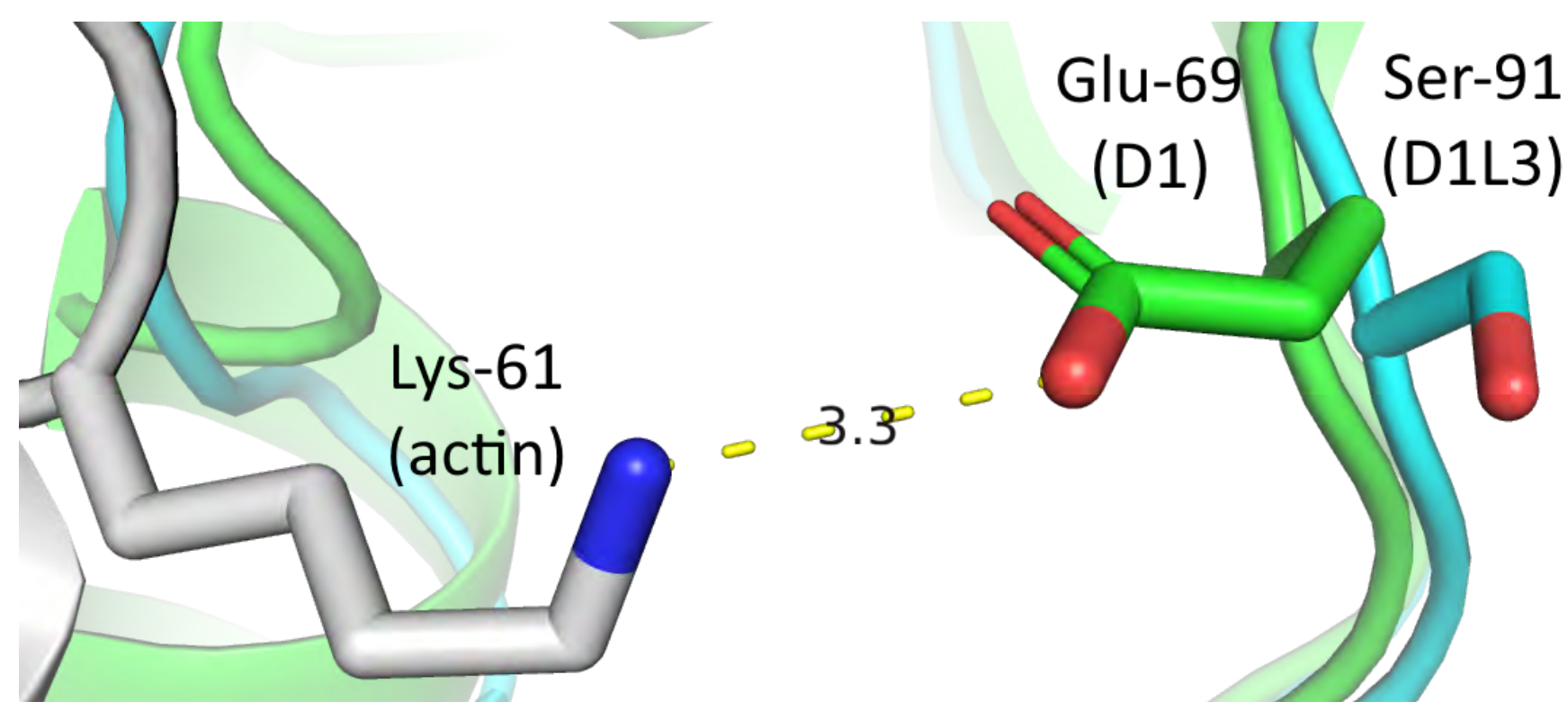

Figure S3. Dnase1 forms a salt bridge to actin between the Dnase1 residue Glu-69 and the actin residue Lys-61. This salt bridge was shown to be a close contact site by cross-linking experiments and the Dnase 1 crystal structure ${ }^{22,69}$. The corresponding residue to Dnase1 Glu-69 on Dnase1L3 is Ser-91. The calculated distance between a Ser at this locus and the Lys-61 of actin would be $>(7 \AA)$. Therefore, it is unlikely that Dnase1L3 could form a productive salt bridge to actin at this locus. 
bioRxiv preprint doi: https://doi.org/10.1101/2022.01.19.476959; this version posted January 20, 2022. The copyright holder for this preprint (which was not certified by peer review) is the author/funder. All rights reserved. No reuse allowed without permission.

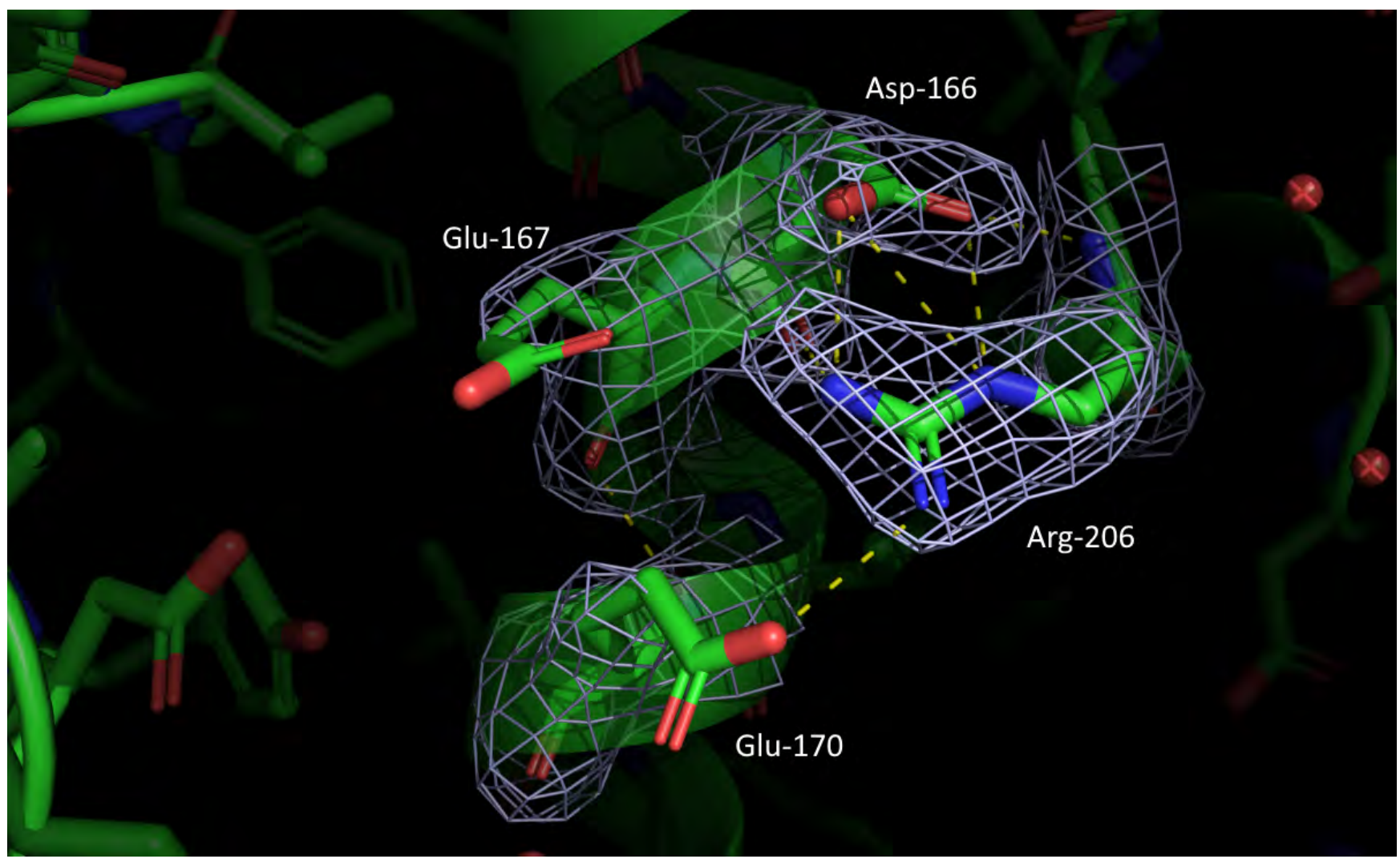

Figure S4. Experimental $\left(2 \mathrm{~F}_{\mathrm{O}}-\mathrm{F}_{\mathrm{C}}\right)$ electron density (contoured at $\left.1 \sigma\right)$ of Dnase $1 \mathrm{~L} 3 \Delta \mathrm{CTD}$ Chain $\mathrm{C}$ showing the salt-bridged network of Arg-206 that stabilizes the conformation of nearby critical residues by tying two helical elements that are close to the active site. The distances indicated are $2.9 \AA$ from each of the carboxylic acid side chain oxygen's of Asp-166 to two of the positively charged amine groups of Arg-206. Arg-206 also positions the terminal amine groups $3.5 \AA$ and $3.1 \AA$ from Glu-167 and Glu-170 respectively. 

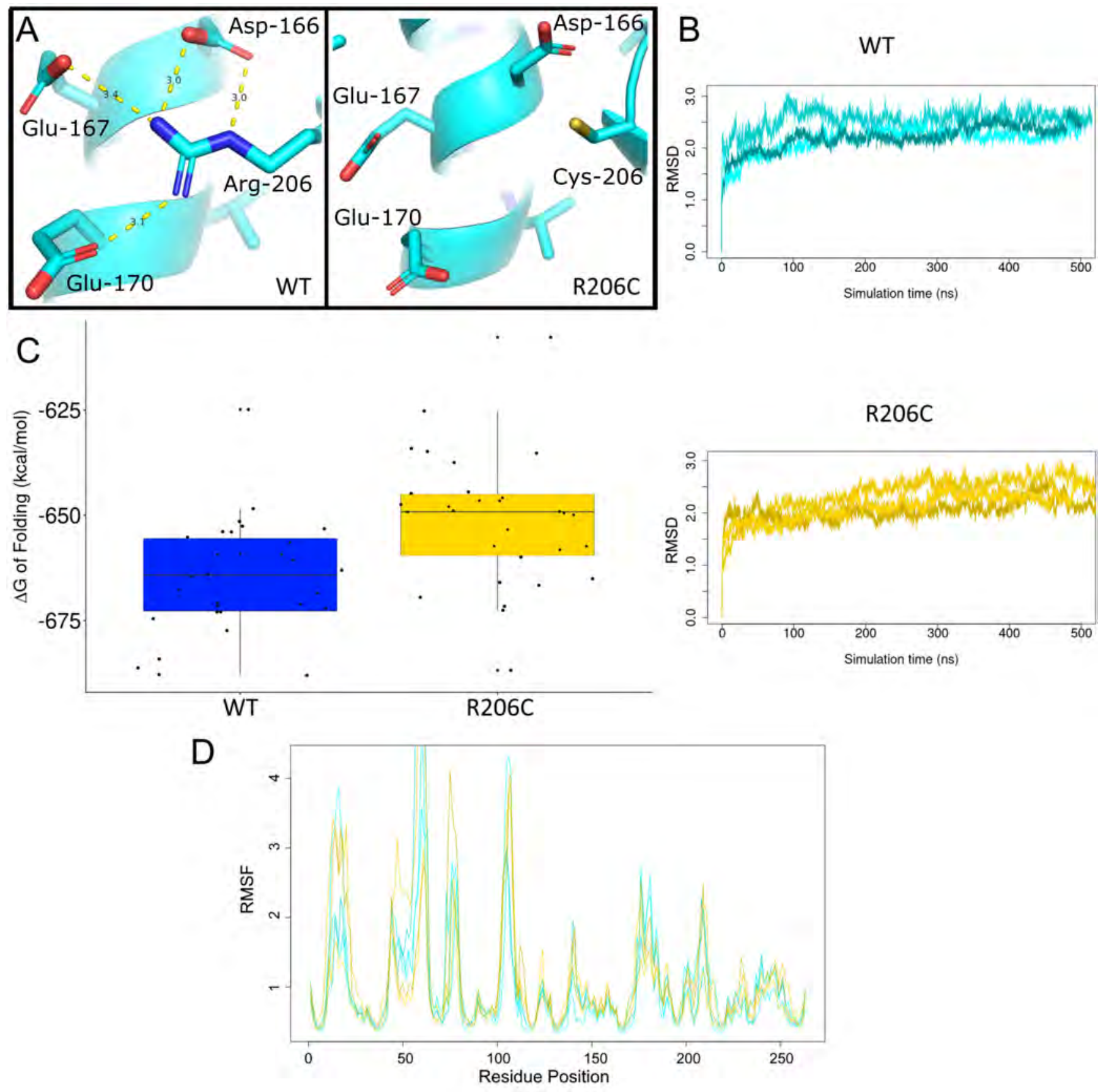

Figure S5. Molecular Dynamics analysis of the R206C mutation. (A) final snapshot from the $500 \mathrm{~ns}$ simulations of either Chain A from the crystal structure of Dnase1L3 with Arg206 (WT) or the R206C mutation. (B) RMSD from three $500 \mathrm{~ns}$ simulations of WT Dnase1L3 (top in blue) and R206C (bottom in yellow).(C) calculated $\Delta \mathrm{G}$ of folding in $\mathrm{kcal} / \mathrm{mol}$ of the WT enzyme and R206C, from 10 snapshots (10 ns apart) of the last $100 \mathrm{~ns}$ of each of the six simulations (three for WT and three for R206C). The free energy of folding was calculated using Rosetta. The difference between WT and the R206C mutation is $14.03 \mathrm{kcal} / \mathrm{mol} \pm 3.79$ (s.d.), $\mathrm{p}$-value $=0.000476$. The less favorable free energy of folding of R206C corresponds to the disruption of three salt bridges (3-5 kcal/mol $)^{70}$, in accordance with our crystal structure. (D) The RMSF of each of the six MD simulations, WT (Arg-206) is shown in blue and R206C is shown in yellow. There is no significant increase in mobility (RMSF) for the loop residues preceding Arg206, when it is modified to a cysteine residue 
bioRxiv preprint doi: https://doi.org/10.1101/2022.01.19.476959; this version posted January 20, 2022. The copyright holder for this preprint (which was not certified by peer review) is the author/funder. All rights reserved. No reuse allowed without permission.

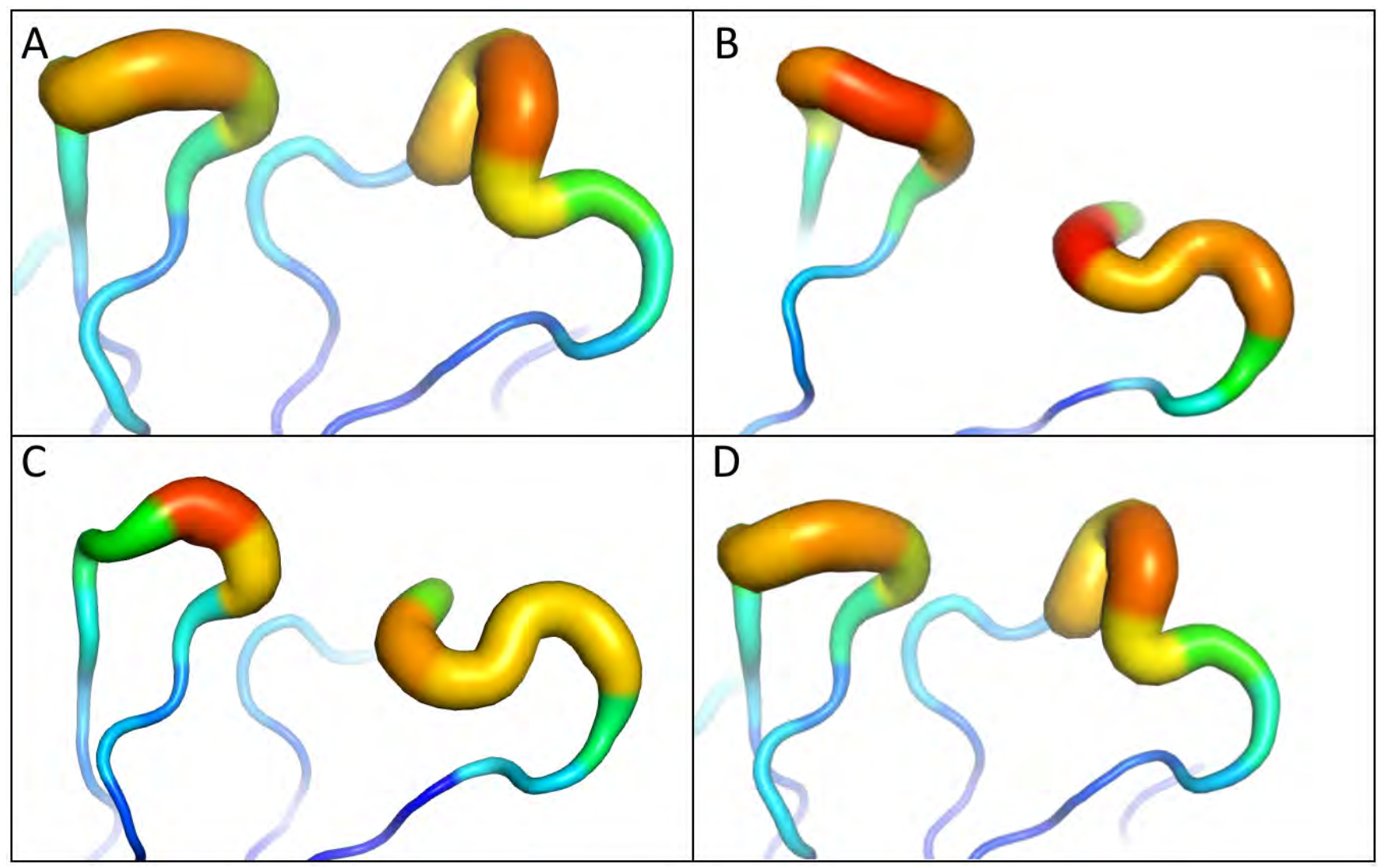

Figure S6. Each of the four chains in the crystallographic asymmetric unit of Dnase1L3 (PDB:7KIU) highlighting the high B-factor around the 'II' divalent cation binding loop. Blue represents a low-B factor region and red amino acids have a relatively high B-factor region. The highly acidic coordinating residues of the 'II' loop are conserved; however, a missing disulfide bridge in Dnase1L3, compared to Dnase1, disrupts divalent cation coordination. Consistent with a non-coordinating highly charged loop region, the 'II' loop is highly flexible and has increased flexibility as measured by the B-factor putty plot across all four chains. 
bioRxiv preprint doi: https://doi.org/10.1101/2022.01.19.476959; this version posted January 20, 2022. The copyright holder for this preprint (which was not certified by peer review) is the author/funder. All rights reserved. No reuse allowed without permission.

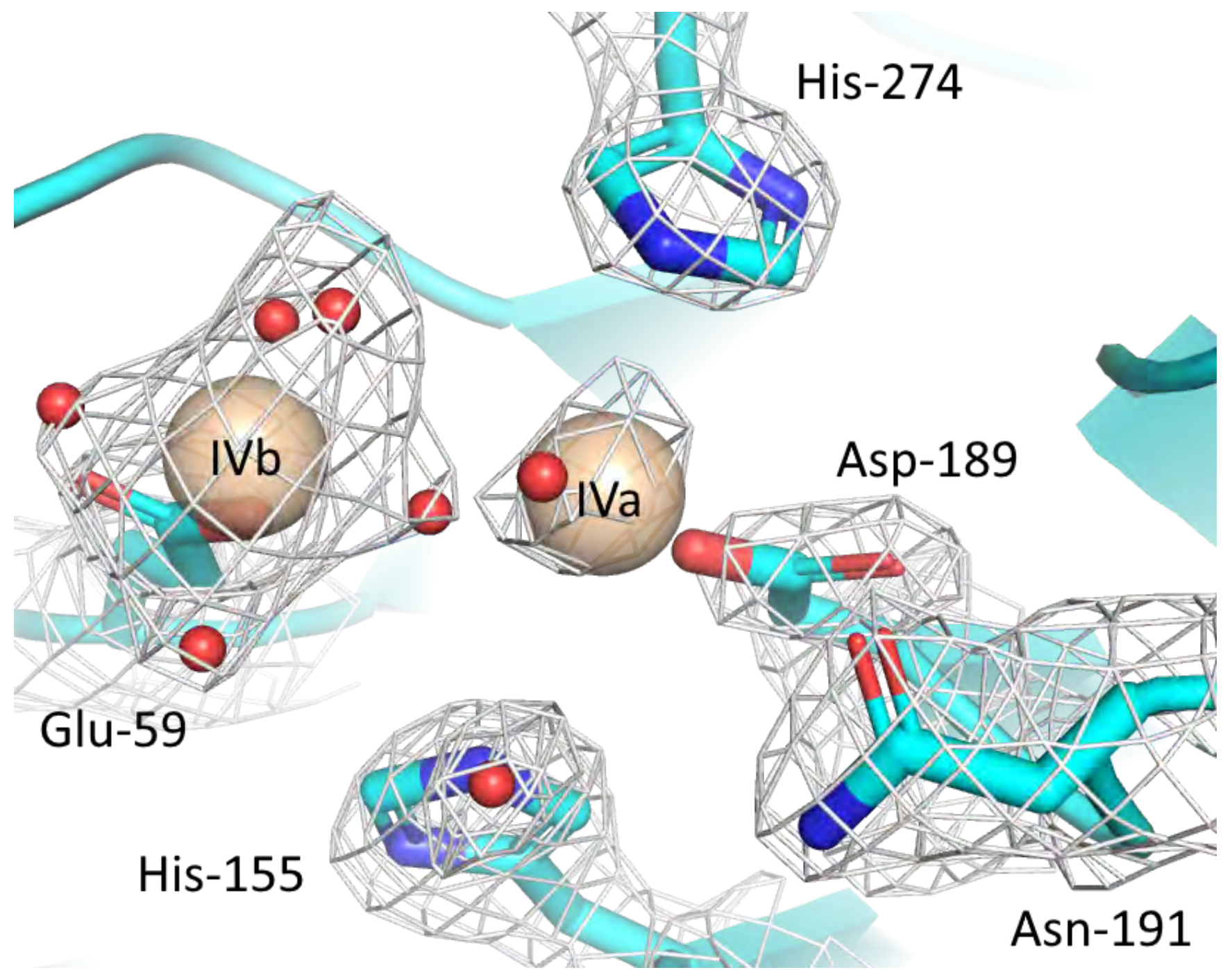

Figure S7. Dnase1L3 crystallized with two $\mathrm{Mg}^{2+}$ in the active site. The $2 \mathrm{~F}_{\mathrm{O}}-\mathrm{F}_{\mathrm{C}}$ density is contoured at $1 \sigma$. The previously observed 'IVb' divalent cation ${ }^{10}$ is coordinated by Glu-59 and five water molecules which are coordinated by Glu-59, Asp-273, and Asn-27. One water molecule is co-coordinated between 'IVb' and 'IVa'. The 'IVa' divalent cation is coordinated by Asp-189, Asn-191, His-274, and three water molecules, one of which is coordinated by His-155 and another is coordinated by Asn-191. The His-274 coordination of 'IVa' was observed to be transient in molecular dynamics simulations. Indeed there is extra density observed between His-274 and 'IVa' that may be an additional coordinating water molecule. 


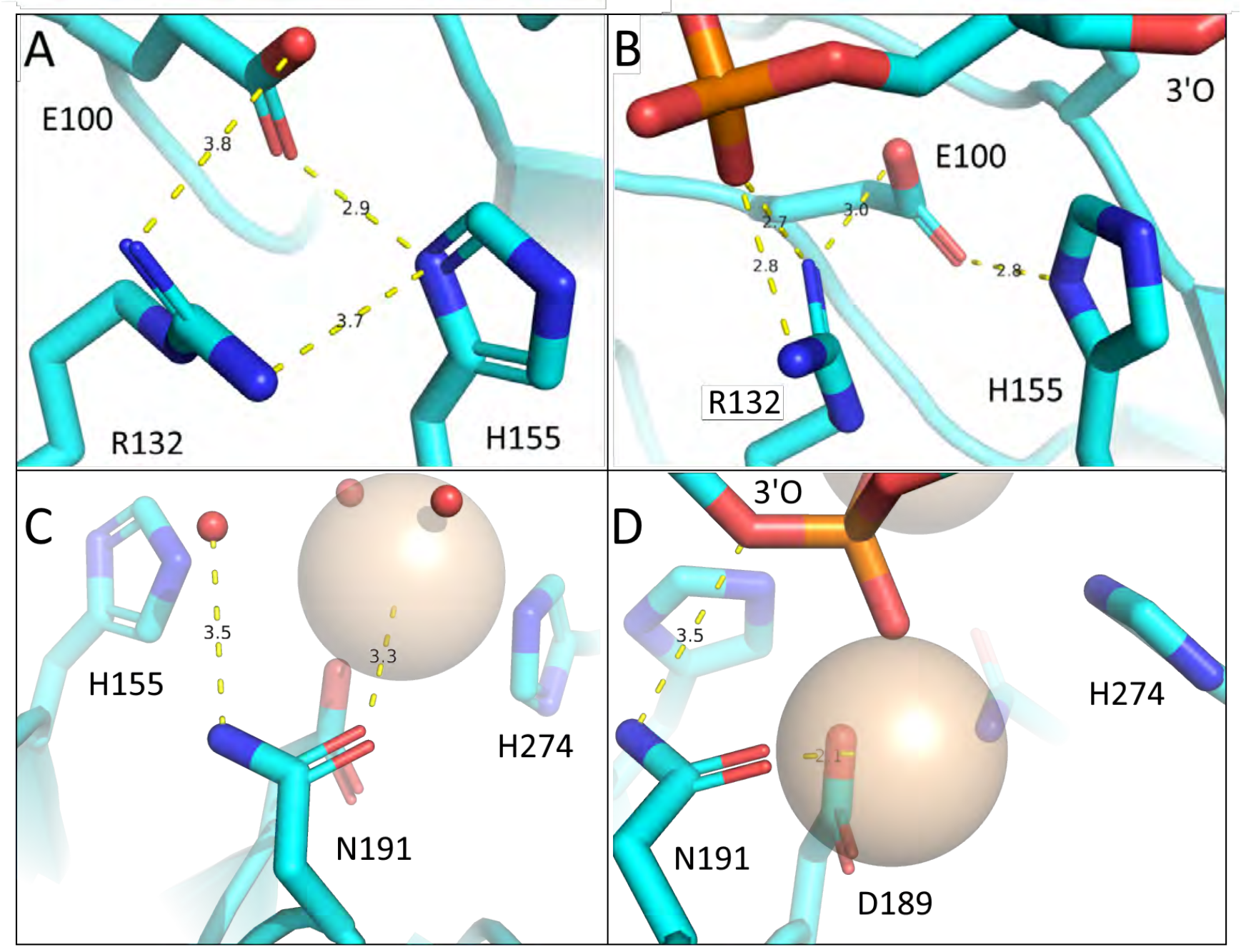

Figure S8. (A) Arg-132 interacts with His-155 in the Dnase1L3 crystal structure in the absence of DNA: According to our MD simulation, in the presence of DNA (B) Arg-132 switches to bind the phosphate backbone and Glu-100 alone coordinates His-155. The Arg-132 substrate switch may prime His-155 to accept a proton, thus regenerating the protonating ${ }^{11}$ His-155. (C and D) Asn-191 coordinates the active site $\mathrm{Mg}^{2+}$ divalent cation 'IVa' in the Dnase1L3 crystal structure (C) and molecular dynamics in the presence of DNA (D). Asn-191 still interacts through the $\delta 2$ amine with the oxygen of a solvent water that roughly corresponds to the location of the 3' oxygen of the scissile phosphate, which is shown coordinated in (D). The red spheres in (C) are crystallographic waters and the tan sphere coordinated by N191 in $\left(\mathbf{C}\right.$ and D) is the ' $\mathrm{IVa}^{\prime} \mathrm{Mg}^{2+}$. 
bioRxiv preprint doi: https://doi.org/10.1101/2022.01.19.476959; this version posted January 20, 2022. The copyright holder for this preprint (which was not certified by peer review) is the author/funder. All rights reserved. No reuse allowed without permission.

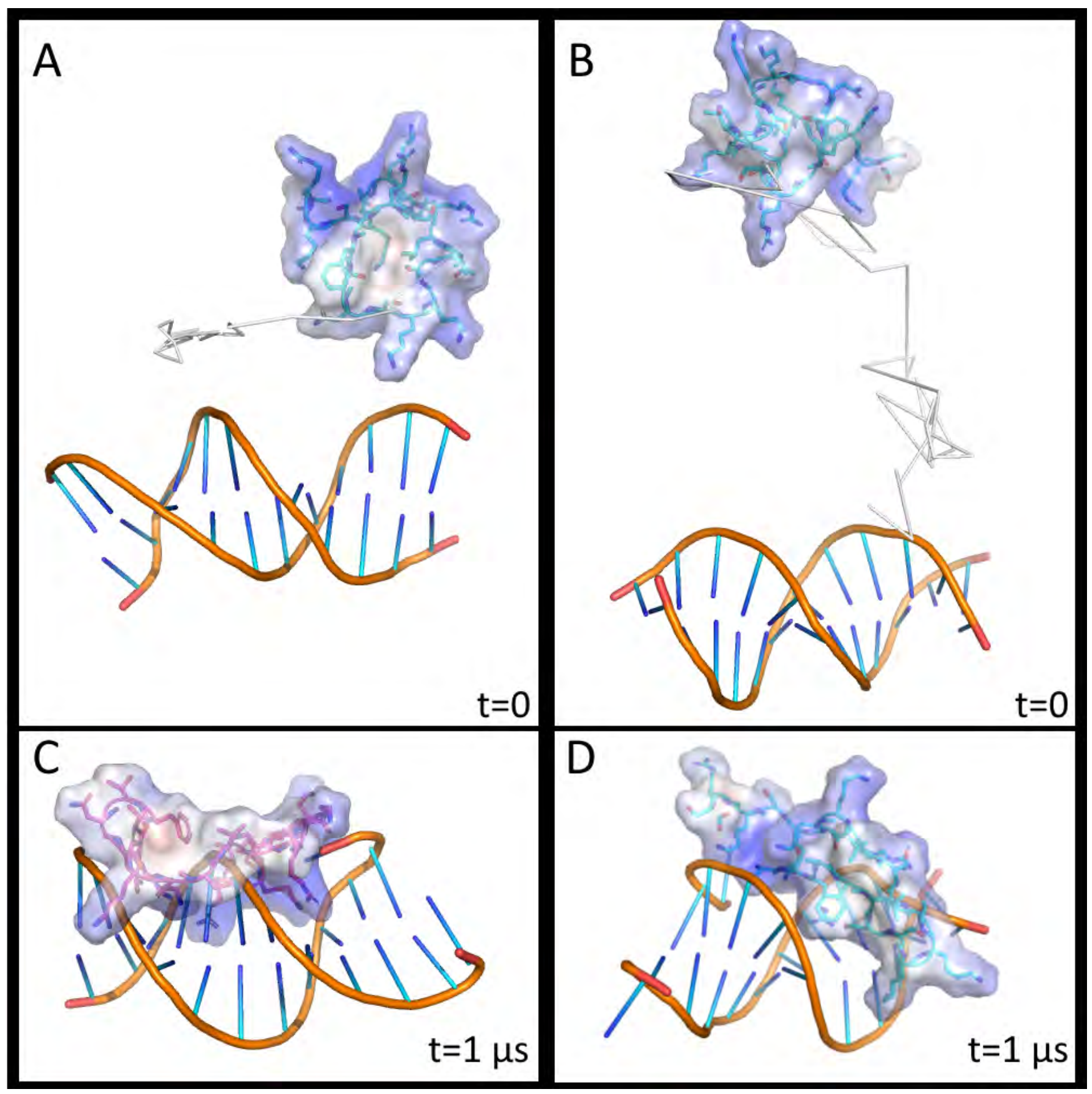

Figure S9. The CTD structure was resolved with solution-state NMR and analyzed in an explicit solvent box with a 16-mer of dsDNA. (A and B) The initial setup for each simulation is shown. The electrostatic potential of the CTD peptide is indicated by the colored volume surrounding the peptide. Blue shading signifies positively-charged potential. The trajectory was traced over the first $12 \mathrm{~ns}$ by plotting the position of a centrally-positioned C- $\alpha$ (Val-294). (C and D) The final configuration of each CTD-DNA simulation at $1 \mu \mathrm{s}$ is shown for the corresponding initial conditions. The CTD does not adopt a distinct secondary structure in the presence of dsDNA, and the two simulations do not converge on a common CTD binding conformation. 